TRANSACTIONS OF THE

AMERICAN MATHEMATICAL SOCIETY

Volume 348, Number 3, March 1996

\title{
EVEN LINKAGE CLASSES
}

\author{
SCOTT NOLLET
}

\begin{abstract}
In this paper we generalize the $\mathcal{E}$ and $\mathcal{N}$-type resolutions used by Martin-Deschamps and Perrin for curves in $\mathbb{P}^{3}$ to subschemes of pure codimension in projective space, and shows that these resolutions are interchanged by the mapping cone procedure under a simple linkage. Via these resolutions, Rao's correspondence is extended to give a bijection between even linkage classes of subschemes of pure codimension two and stable equivalence classes of reflexive sheaves $\mathcal{E}$ satisfying $H_{*}^{1}(\mathcal{E})=0$ and $\mathcal{E} x t^{1}\left(\mathcal{E}^{\vee}, \mathcal{O}\right)=0$. Further, these resolutions are used to extend the work of Martin-Deschamps and Perrin for Cohen-Macaulay curves in $\mathbb{P}^{3}$ to subschemes of pure codimension two in $\mathbb{P}^{n}$. In particular, even linkage classes of such subschemes satisfy the Lazarsfeld-Rao property and any minimal subscheme for an even linkage class links directly to a minimal subscheme for the dual class.
\end{abstract}

\section{INTRODUCTION}

Much progress has been made in the area of linkage theory for subschemes of $\mathbb{P}^{n}$ over the past 15 years, but most of the work has dealt exclusively with locally Cohen-Macaulay (CM) subschemes. This is somewhat restrictive, since linkage applies more generally to equidimensional subschemes without embedded components. In particular, linkage techniques apply to integral subschemes, which need not be CM. The purpose of this paper is to extend several standard theorems for $\mathrm{CM}$ subschemes to the more general setting.

In the first section, we generalize the $\mathcal{E}$ and $\mathcal{N}$-type resolutions used by MartinDeschamps and Perrin [10] to subschemes in $\mathbb{P}^{n}$ of codimension $r>1$. We show that the equidimensional subschemes of $\mathbb{P}^{n}$ having no embedded components can be characterized by an algebraic condition on their $\mathcal{E}$-type resolutions (see Corollary 1.10). For subschemes which are linked, we show that the mapping cone procedure [14, Proposition 2.5] interchanges $\mathcal{E}$ and $\mathcal{N}$-type resolutions for the two subschemes.

It was a great discovery of Rao that the even linkage classes of locally CohenMacaulay codimension two subschemes of $\mathbb{P}^{n}$ are in bijective correspondence with stable equivalence classes of vector bundles $\mathcal{F}$ on $\mathbb{P}^{n}$ which satisfy $H_{*}^{1}(\mathcal{F})=0$ [16]. In the second section of this paper, we extend Rao's correspondence to show that the even linkage classes of pure codimension two subschemes of $\mathbb{P}^{n}$ are in bijective correspondence with stable equivalence classes of reflexive sheaves $\mathcal{F}$ on $\mathbb{P}^{n}$ which satisfy $H_{*}^{1}(\mathcal{F})=0$ and $\mathcal{E} x t^{1}\left(\mathcal{F}^{\vee}, \mathcal{O}\right)=0$ (see Theorem 2.11). Since $\mathcal{E} x t^{1}\left(\mathcal{F}^{\vee}, \mathcal{O}\right)=0$ for any vector bundle $\mathcal{F}$, this gives a generalization of vector bundle.

Received by the editors March 6, 1995

1991 Mathematics Subject Classification. Primary 14M06; Secondary 14M12, 13C40.

Key words and phrases. Even linkage classes, Lazarsfeld-Rao property, Rao's correspondence. 
Perhaps the most important theorem about even linkage classes of locally CohenMacaulay codimension two subschemes of $\mathbb{P}^{n}$ is the fact that they satisfy the Lazarsfeld-Rao property (see [1]; the case of curves in $\mathbb{P}^{3}$ was done in [10]). By replacing vector bundles with reflexive sheaves, the proof in [10] goes through with minor modifications (see Theorem 3.26). The concept of domination is used to simplify the statement of the main theorem.

The last two sections of this paper deal only with subschemes of codimension two. It would be interesting to know if Rao's correspondence (section two) can be generalized in some way via the resolutions used in section one. If this situation were better understood, then perhaps the methods of section three might be used to investigate whether the Lazarsfeld-Rao property holds for even linkage classes of subschemes of codimension $>2$.

I'd like to thank Robin Hartshorne and Juan Migliore for editorial suggestions, especially regarding the first section. I appreciate personal communications with A. P. Rao about the details of his work. Finally, I'd like to thank Martin-Deschamps and Perrin for their book [10]. The generality with which they handle the fourth chapter saved this author a great deal of work.

\section{Two Resolutions}

In this section we generalize the $\mathcal{E}$-type and $\mathcal{N}$-type resolutions for curves found in [10] to subschemes of pure codimension $r$ in $\mathbb{P}^{n}$ (in other words, equidimensional subschemes with no embedded components). We show in this broader setting that the mapping cone procedure [14, Proposition 2.5] still works and interchanges these two types of resolutions for subschemes which are linked. Before getting on to these results we briefly recall the definition of linkage and some of the first properties. The main reference for this is [7], where the foundations of linkage theory have been rewritten in the more conceptual context of generalized divisors.

Definition 1.1. Let $V_{1}, V_{2}$ be subschemes of codimension $r$ in $\mathbb{P}^{n}$. We say that $V_{1}$ is linked to $V_{2}$ by the complete intersection $X$ if $X$ is a global complete intersection of codimension $r$ containing $V_{1}$ and $V_{2}$ in such a way that

$$
\begin{aligned}
& \mathcal{I}_{V_{2}, X} \cong \mathcal{H o m}\left(\mathcal{O}_{V_{1}}, \mathcal{O}_{X}\right), \\
& \mathcal{I}_{V_{1}, X} \cong \mathcal{H o m}\left(\mathcal{O}_{V_{2}}, \mathcal{O}_{X}\right) .
\end{aligned}
$$

Proposition 1.2. Linkage enjoys the following properties:

1. If $V_{1}$ is linked to $V_{2}$ by the complete intersection $X$, then $V_{2}$ is linked to $V_{1}$ by $X$.

2. If $V_{1} \subset \mathbb{P}^{n}$ is of pure codimension $r$ and is contained in a complete intersection $X$ of codimension $r$, then there exists a unique subscheme $V_{2} \subset \mathbb{P}^{n}$ of pure codimension $r$ such that $V_{2}$ is linked to $V_{1}$ by $X$.

3. If $V_{1}$ is linked to $V_{2}$ by $X$, then either both $V_{1}$ and $V_{2}$ are of pure codimension $r$ in $\mathbb{P}^{n}$, or one of them is empty.

4. If $V_{1}$ is linked to $V_{2}$, then $V_{1}$ is locally Cohen-Macaulay if and only if $V_{2}$ is locally Cohen-Macaulay.

Proof. Part (3) is proved in [13, Proposition 4.2 (2)], and credited to Schwartau's thesis, although it was surely known before then. Parts (1), (2) and (4) can be found 
in Propositions 4.1 and 4.2 of [7]. In the latter paper, the definition of linkage is different, but is proved to be equivalent to the usual definition.

Remark 1.3. Part (4) shows that linkage preserves the property of being locally Cohen-Macaulay. In the literature, many papers only apply linkage theory to locally Cohen-Macaulay schemes. The purpose of this paper is to generalize many of these results to subschemes which are not locally Cohen-Macaulay.

Definition 1.4. A sheaf $\mathcal{F}$ on $\mathbb{P}^{n}$ is called dissocié if it is a direct sum of line bundles.

Definition 1.5. If $V \subset \mathbb{P}^{n}$ is a subscheme of codimension $r$, then an $\mathcal{E}$-type resolution for $V$ is an exact sequence

$$
0 \rightarrow \mathcal{E} \rightarrow \mathcal{Q}_{r-1} \rightarrow \mathcal{Q}_{r-2} \rightarrow \ldots \rightarrow \mathcal{Q}_{1} \rightarrow \mathcal{O}\left(\rightarrow \mathcal{O}_{V} \rightarrow 0\right)
$$

such that $\mathcal{Q}_{i}$ is dissocié for $0<i<r$ and $H_{*}^{i}(\mathcal{E})=0$ for $0<i<r$.

Remark 1.6. The condition that $H_{*}^{i}(\mathcal{E})=0$ for $0<i<r$ can be interpreted as follows. Let $F_{i}$ denote the kernel of the map from $\mathcal{Q}_{i}$. Breaking the resolution into short exact sequences shows that for each $0 \leq k<r$, the graded modules $H_{*}^{i}\left(F_{i+k}\right)$ are all isomorphic for $1 \leq i<r-k$. Thus the condition $H_{*}^{i}(\mathcal{E})=0$ for $0<i<r$ is equivalent to the condition that $H_{*}^{1}\left(F_{i}\right)=0$ for $1 \leq i<r$, which is equivalent to saying that applying $H_{*}^{0}$ to the resolution gives a resolution of graded $S$-modules for $I_{V}$. In particular, every subscheme $V \subset \mathbb{P}^{n}$ has an $\mathcal{E}$-type resolution, which is obtained by sheafifying a graded free resolution of $I_{V}$ and taking $\mathcal{E}$ to be the kernel of the $r$ th map.

Remark 1.7. Since $V$ is a scheme of dimension $n-r$, the local depth of $\mathcal{O}_{V}$ is $\leq n-r$, hence the local cohomological dimension of $\mathcal{O}_{V}$ is always $\geq r$ by the theorem of Auslander and Buchsbaum. This shows that $\mathcal{E} \neq 0 . \mathcal{E}$ is locally free if and only if $V$ is locally Cohen-Macaulay, because both these conditions are equivalent to the condition that the local cohomological dimension of $V$ is equal to $r$.

Proposition 1.8. Let $V \subset \mathbb{P}^{n}$ be a subscheme of codimension $r$ which is contained in a complete intersection $X$ of $r$ hypersurfaces of degrees $\left\{d_{i}\right\}$. Let $G$ be an $\mathcal{E}$-type resolution for $V$ and let $F$ denote the Koszul resolution for $X$. Then there is a morphism of complexes $\alpha: F \rightarrow G$ induced by the inclusion $\mathcal{I}_{X} \rightarrow \mathcal{I}_{V}$, and the mapping cone of $\alpha^{\vee}\left(-\sum d_{i}\right)$ gives a resolution for $\mathcal{O}_{W}$, where $W$ is the subscheme of $X$ defined by $\mathcal{I}_{W, X} \cong \mathcal{H} m_{\mathcal{O}_{X}}\left(\mathcal{O}_{V}, \mathcal{O}_{X}\right)$.

Proof. In the context of Definition 1.5 , we identify $G_{0}$ with $\mathcal{O}$ and $G_{r}$ with $\mathcal{E}$. By Remark 1.6, the condition $H_{*}^{i}(\mathcal{E})=0$ for $0<i<r$ is equivalent to the condition that applying $H_{*}^{0}$ to the resolution gives an exact sequence of graded $S$-modules. In particular, we can find a morphism from the Koszul resolution of graded $S$-modules to the $\mathcal{E}$-type resolution, and sheafifying gives the desired morphism $\alpha$.

$\mathcal{E} x t$ can be computed from locally free resolutions [5, III, Proposition 6.5], so we have isomorphisms $H_{i}\left(F^{\vee}\right) \cong \mathcal{E} x t^{i}\left(\mathcal{O}_{X}, \mathcal{O}\right)$. These $\mathcal{E} x t$ s vanish for $i<r$ by Ischebeck's theorem [12, Theorem 17.1], because $X$ is of codimension $r$. Now set $d=$ $\sum d_{i}$ and let $C$ denote the mapping cone to $\alpha^{\vee}(-d)$. In view of the vanishings above, the long exact homology sequence associated to $C$ gives isomorphisms $H_{i}(C) \cong$ $H_{i}\left(G^{\vee}(-d)\right)$ for $0 \leq i<r$ and an exact sequence

$$
0 \rightarrow H_{r}(C) \rightarrow H_{r}\left(G^{\vee}(-d)\right) \rightarrow H_{r}\left(F^{\vee}(-d)\right) \rightarrow H_{r+1}(C) \rightarrow 0 .
$$


Now let $L$ be a dissocié resolution for $\mathcal{O}_{V}$ which agrees with $G$ up to the $(r-1)$ st step, so that $L_{\geq r}$ is a resolution for $G_{r}=\mathcal{E}, L_{0}=\mathcal{O}$ and $L_{i}=$ $\mathcal{Q}_{i}$ for $0<i<r$. Clearly the morphism $\alpha$ can be factored through the complex $L$ as $F \stackrel{\gamma}{\rightarrow} L \stackrel{\beta}{\longrightarrow} G$. Since $\gamma$ is a morphism of locally free resolutions of $\mathcal{O}_{V}$ and $\mathcal{O}_{X}$, the induced homology map $H_{r}\left(\gamma^{\vee}(-d)\right)$ is the canonical map $\mathcal{E} x t^{r}\left(\mathcal{O}_{V}, O\right)(-d) \rightarrow \mathcal{E} x t^{r}\left(\mathcal{O}_{X}, O\right)(-d)$. Since the functor $\mathcal{E} x t_{\mathcal{O}}^{r}(*, \mathcal{O})(-d)$ on the category of $\mathcal{O}_{X}$-modules is isomorphic to the functor $\mathcal{H o m}_{\mathcal{O}_{X}}\left(*, \mathcal{O}_{X}\right)$, this map is identified with the inclusion $\mathcal{H o m}_{\mathcal{O}_{X}}\left(\mathcal{O}_{V}, \mathcal{O}_{X}\right) \hookrightarrow \mathcal{H o m}_{\mathcal{O}_{X}}\left(\mathcal{O}_{X}, \mathcal{O}_{X}\right)$. It follows that the map $H_{r}\left(L^{\vee}(-d)\right) \rightarrow H_{r}\left(F^{\vee}(-d)\right)$ is injective and has $\mathcal{O}_{W}$ as cokernel. To finish the proof of the proposition, it suffices to show that the homology maps $H_{i}\left(G^{\vee}(-d)\right) \rightarrow H_{i}\left(L^{\vee}(-d)\right)$ are isomorphisms for $0 \leq i \leq r$.

For $0 \leq i<r-1$ it is clear that $H_{i}\left(\beta^{\vee}(-d)\right)$ is an isomorphism, because $\beta$ is an isomorphism in these degrees. For $i=r-1$ and $r$, we have a fragment of the commutative diagram corresponding to $\beta^{\vee}$

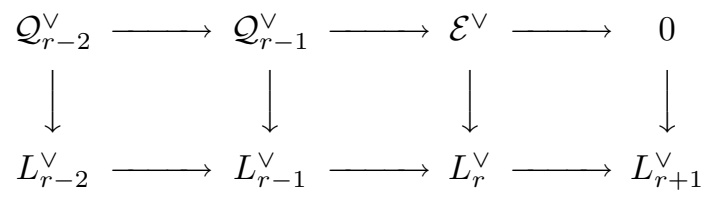

The two vertical maps on the left are isomorphisms by our choice of $L$. Because $L_{\geq r}$ is a resolution for $\mathcal{E}$ and dualizing is left exact, we see that $\mathcal{E}^{\vee}$ is precisely the kernel of the map $L_{r}^{\vee} \rightarrow L_{r+1}^{\vee}$. Using this, a little diagram chase shows that the homology maps $H\left(\mathcal{Q}_{r-1}^{\vee}\right) \rightarrow H\left(L_{r-1}^{\vee}\right)$ and $H\left(\mathcal{E}^{\vee}\right) \rightarrow H\left(L_{r}^{\vee}\right)$ are isomorphisms.

Remark 1.9. If we skip past the first paragraph, the proof above is local commutative algebra. The local statement proved is as follows: Let $(A, m)$ be a regular local ring and let $I \subset A$ be an ideal which contains a regular sequence $\left\{a_{i}\right\}$ of length $r$. Let $J$ be the ideal generated by the $a_{i}$. If $C$ is a resolution for $A / I$ of the form

$$
0 \rightarrow E \rightarrow F_{r-1} \rightarrow F_{r-2} \rightarrow \ldots \rightarrow F_{1} \rightarrow A(\rightarrow A / I \rightarrow 0)
$$

with $F_{i}$ free, $D$ is the Koszul complex for $A / J$, and $\alpha$ is any morphism $D \rightarrow C$ induced by the map $A / J \rightarrow A / I$, then the mapping cone of $\alpha^{\vee}$ gives a resolution for $A / K$, where $K$ is the ideal containing $J$ defined by $K / J \cong \mathcal{H o m}(A / I, A / J)$.

Corollary 1.10. If $V \subset \mathbb{P}^{n}$ is a subscheme of pure codimension $r$ with $\mathcal{E}$-type resolution as in Definition 1.5 and $V$ links to $W$ via a complete intersection $X$ of hypersurfaces with degrees summing to $d$ and having Koszul resolution $F$, then $W$ has a resolution

$$
0 \rightarrow \mathcal{P}_{r-1} \rightarrow \mathcal{P}_{r-2} \rightarrow \ldots \rightarrow \mathcal{P}_{1} \rightarrow \mathcal{N} \rightarrow \mathcal{O} \rightarrow \mathcal{O}_{W} \rightarrow 0
$$

where $\mathcal{P}_{r-1}=\mathcal{Q}_{1}^{\vee}(-d), \mathcal{P}_{i}=\mathcal{Q}_{r-i}^{\vee}(-d) \oplus F_{r-i-1}^{\vee}(-d)$ for $1 \leq i<r-1$ and $\mathcal{N}=\mathcal{E}^{\vee}(-d) \oplus F_{r-1}^{\vee}(-d)$.

Proof. In this case, the scheme $W$ linked to $V$ satisfies $\mathcal{I}_{W, X} \cong \mathcal{H o m}_{\mathcal{O}_{X}}\left(\mathcal{O}_{V}, \mathcal{O}_{X}\right)$ by the definition of linkage. Hence it is the scheme $W$ of the previous proposition. The mapping cone obtained in the previous proposition has form almost like the one given above, except that the summands $\mathcal{O}^{\vee}(-d)$ at the left end have been split off (which is possible because the induced map is the identity). 
Proposition 1.11. Let $V \subset \mathbb{P}^{n}$ be a subscheme which is contained in the complete intersection $X$ of $r$ hypersurfaces of degrees $\left\{d_{i}\right\}$. Let $G$ be a resolution for $\mathcal{O}_{V}$ of the form

$$
0 \rightarrow \mathcal{P}_{r-1} \rightarrow \mathcal{P}_{r-2} \rightarrow \ldots \rightarrow \mathcal{P}_{1} \rightarrow \mathcal{N} \rightarrow \mathcal{O}\left(\rightarrow \mathcal{O}_{V} \rightarrow 0\right)
$$

where $\mathcal{P}_{i}$ are dissocié. If $F$ denotes the Koszul resolution for $X$, then there is a morphism $\alpha: F \rightarrow G$ induced by the inclusion $\mathcal{I}_{X} \rightarrow \mathcal{I}_{V}$. If $W$ is the subscheme of $X$ defined by $\mathcal{I}_{W, X} \cong \mathcal{H} m_{\mathcal{O}_{X}}\left(\mathcal{O}_{V}, \mathcal{O}_{X}\right)$, then the mapping cone of the morphism $\alpha^{\vee}\left(-\sum d_{i}\right)$ gives a resolution of $\mathcal{O}_{W}$ if and only if $\mathcal{E} x t^{i}(\mathcal{N}, \mathcal{O})=0$ for $1 \leq i<r$.

Proof. Let $R_{i}$ denote the image of $\mathcal{P}_{i}$ under the maps for the resolution for $\mathcal{O}_{V}$. We can break the resolution for $\mathcal{O}_{V}$ into short exact sequences (with dissocié sheaves in the middle). The long exact cohomology sequence shows that for each $0<i<r$ we have that $H_{*}^{1}\left(R_{i}\right) \cong H_{*}^{r-i}\left(R_{r-1}\right)$, but $R_{r-1} \cong \mathcal{P}_{r-1}$ is dissocié, hence all these graded $S$-modules are zero. It follows that when we apply $H_{*}^{0}$ to $G$ we get an exact sequence of graded $S$-modules. We can find a resolution for $I_{V}$ consisting of free graded $S$-modules which maps surjectively to this exact sequence on each component. Sheafifying gives a dissocié resolution $L$ for $\mathcal{O}_{V}$ which maps surjectively to the resolution that we started with. The inclusion of total ideals $I_{X} \subset I_{V}$ induces a morphism from the Koszul resolution of graded $S$-modules for $\mathcal{O}_{X}$ to the graded free resolution $H_{*}^{0}(L)$. Thus we obtain a morphism $\alpha: F \rightarrow G$ of complexes which factors through $L$ as

$$
F \stackrel{\gamma}{\rightarrow} L \stackrel{\beta}{\longrightarrow} G
$$

Set $d=\sum d_{i}$ and let $C$ be the mapping cone of $\alpha^{\vee}(-d)$. We consider the long exact homology sequence associated to the mapping cone. As in Proposition 1.8 , we use the fact that $X$ is of codimension $r$ to see that $H_{i}\left(F^{\vee}(-d)\right)=0$ for $0<i<r$. It follows from the long exact homology sequence that we have isomorphisms $H_{i}(C) \cong H_{i}\left(G^{\vee}(-d)\right)$ for $0 \leq i<r$ and an exact sequence

$$
0 \rightarrow H_{r}(C) \rightarrow H_{r}\left(G^{\vee}(-d)\right) \rightarrow H_{r}\left(F^{\vee}(-d)\right) \rightarrow H_{r+1}(C) \rightarrow 0 .
$$

Computing $\mathcal{E} x t$ from the locally free resolution $L$ gives

$$
H_{r}\left(L^{\vee}(-d)\right)=\mathcal{E} x t^{r}\left(\mathcal{O}_{V}, \mathcal{O}\right)(-d)=\mathcal{H o m}_{\mathcal{O}_{X}}\left(\mathcal{O}_{V}, \mathcal{O}_{X}\right)
$$

and this last sheaf is isomorphic to $\mathcal{I}_{W, X}$ by definition of $W$. The map from this sheaf to $H_{r}\left(F^{\vee}(-d)\right) \cong \mathcal{O}_{X}$ is the canonical inclusion because the functor $\mathcal{E} x t^{r}(*, \mathcal{O})(-d)$ is naturally isomorphic to the functor $\mathcal{H o m}_{\mathcal{O}_{X}}\left(*, \mathcal{O}_{X}\right)$ on the category of $\mathcal{O}_{X}$-modules. It follows that the mapping cone of $\alpha^{\vee}(-d)$ is a resolution for $\mathcal{O}_{W}$ if and only if the homology map $H_{i}\left(\beta^{\vee}\right)$ is an isomorphism for $0 \leq i \leq r$.

Now we compare the homology of $L^{\vee}$ and $G^{\vee}$. Let $K$ be the kernel of the map of complexes $\beta$. Since we chose $L$ with $L_{0}=\mathcal{O}$, we have $K_{0}=0$. In dualizing the exact sequence of complexes we get a commutative diagram

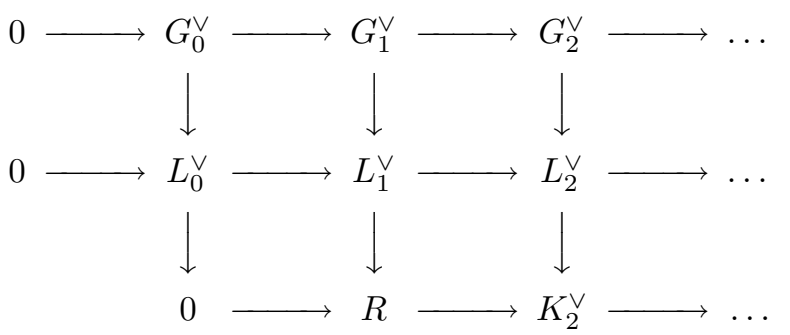


where $R$ denotes the image of the morphism $L_{1}^{\vee} \rightarrow K_{1}^{\vee}$. For $i>1, K_{i}$ is the kernel of the surjection $L_{i} \rightarrow G_{i}$ of bundles, hence the $K_{i}$ are locally free and the $i$ th column of the diagram is a short exact sequence. Clearly the first two columns are exact (the second by choice of $R$ ), so in fact all the columns are exact and we have an associated long exact homology sequence. Since $G_{i}=0$ (and hence $H_{i}\left(G^{\vee}\right)=0$ ) for $i>r$, this long exact sequence shows that $H_{i}\left(\beta^{\vee}\right)$ is an isomorphism for $0 \leq i \leq r$ if and only if the homology $H_{i}$ of the bottom complex is zero for $i \leq r$.

Noting that $R$ is a submodule of $K_{1}^{\vee}$, we have that $H(R)=0$ in any event. Since dualizing is left exact, we see that $H\left(K_{2}^{\vee}\right)=0 \Longleftrightarrow R=K_{1}^{\vee}$. By definition of $R$, this happens $\Longleftrightarrow L_{1}^{\vee} \rightarrow K_{1}^{\vee}$ is surjective. The cokernel of this map is $\mathcal{E} x t^{1}(\mathcal{N}, \mathcal{O})$, because $G_{1}=\mathcal{N}$ and $L_{1}$ is locally free. It follows that $H\left(K_{2}^{\vee}\right)=$ $0 \Longleftrightarrow \mathcal{E} x t^{1}(\mathcal{N}, \mathcal{O})=0$. Finally, since $K_{1}$ is the kernel of the map $L_{1} \rightarrow \mathcal{N}$ we see that

$$
\ldots \rightarrow K_{3} \rightarrow K_{2} \rightarrow L_{1}
$$

is a locally free resolution for $\mathcal{N}$. Since $\mathcal{E} x t$ can be computed from such resolutions [5, III, Proposition 6.5], we see that $H_{i}\left(K^{\vee}\right) \cong \mathcal{E} x t^{i-1}(\mathcal{N})$ for $i>1$. It follows that $H_{i}\left(K^{\vee}\right)=0$ for $2<i \leq r$ if and only if $\mathcal{E} x t^{i}(\mathcal{N})=0$ for $1<i \leq r-1$. Combining the conclusions of this paragraph shows that the homology $H_{i}$ of the bottom complex is exact for $i \leq r \Longleftrightarrow \mathcal{E} x t^{i}(\mathcal{N})=0$ for $1 \leq i<r$. This proves the proposition.

Remark 1.12. Again we have proved a local algebra result: Let $(A, m)$ be a regular local ring, and let $I$ be an ideal which contains a regular sequence $\left\{a_{i}\right\}$ of length $r$ which generates the ideal $J$. Let $K$ be the ideal containing $J$ defined by the condition $K / J \cong \mathcal{H o m}(A / I, A / J)$, and let $C$ be a resolution

$$
0 \rightarrow F_{r-1} \rightarrow F_{r-2} \rightarrow \ldots \rightarrow F_{1} \rightarrow N \rightarrow A(\rightarrow A / I \rightarrow 0)
$$

for $A / I$ with $F_{i}$ free. If $\alpha$ is a morphism from the Koszul resolution of $A / J$ to $C$ induced by the surjection $A / J \rightarrow A / I$, then the mapping cone of $\alpha^{\vee}$ gives a resolution for $A / K$ if and only if $\mathcal{E} x t^{i}(N, A)=0$ for $0<i<r$.

Definition 1.13. If $V \subset \mathbb{P}^{n}$ is a subscheme of codimension $r$, then an $\mathcal{N}$-type resolution for $\mathcal{O}_{V}$ is an exact sequence

$$
0 \rightarrow \mathcal{P}_{r-1} \rightarrow \mathcal{P}_{r-2} \rightarrow \ldots \rightarrow \mathcal{P}_{1} \rightarrow \mathcal{N} \rightarrow \mathcal{O}\left(\rightarrow \mathcal{O}_{V} \rightarrow 0\right)
$$

where $\mathcal{P}_{i}$ are dissocié, $\mathcal{N}$ is reflexive, $\mathcal{E} x t^{i}(\mathcal{N}, \mathcal{O})=0$ for $0<i<r$ and $H_{*}^{i}\left(\mathcal{N}^{\vee}\right)=0$ for $0<i<r$.

Remark 1.14. This looks like a complicated definition, but it is based on a simple idea. We want an $\mathcal{N}$-type resolution for a scheme $V$ to have the property that if $V$ is linked to $W$ by a complete intersection $X$, then the mapping cone should give an $\mathcal{E}$-type resolution for $W$. In view of Proposition 1.11 and the definition of $\mathcal{E}$-type resolution, that is exactly what an $\mathcal{N}$-type resolution does.

Remark 1.15. In Martin-Deschamps and Perrin's work with curves in $\mathbb{P}^{3}$ [10], the definition of $\mathcal{N}$-type resolution is similar, except that instead of the condition that $H_{*}^{1}\left(\mathcal{N}^{\vee}\right)=0$, they have the condition that $H_{*}^{2}(\mathcal{N})=0$. In their situation, the reflexive sheaf $\mathcal{N}$ is locally free (because they only dealt with locally Cohen-Macaulay subschemes), so these conditions are equivalent by Serre duality. 
Proposition 1.16. Let $V \subset \mathbb{P}^{n}$ be of pure codimension $r$ with $\mathcal{E}$-type resolution

$$
0 \rightarrow \mathcal{E} \rightarrow \mathcal{Q}_{r-1} \rightarrow \ldots \rightarrow \mathcal{Q}_{1} \stackrel{\pi}{\longrightarrow} \mathcal{O}\left(\rightarrow \mathcal{O}_{V} \rightarrow 0\right)
$$

Then $\mathcal{E} x t^{i}\left(\mathcal{E}^{\vee}, \mathcal{O}\right)=0$ for $0<i<r$.

Proof. The idea here is simple. We first use a complete intersection $X$ to link $V$ to another scheme $W$, and use Proposition 1.8 to get a resolution for $W$. Then we use a carefully chosen morphism of complexes from the Koszul complex for $\mathcal{O}_{X}$ to this resolution, and compute the cone of the dual of this morphism. By the choice of morphism, we can cancel some redundant factors and are left with the $\mathcal{E}$-type resolution that we started with. Applying Proposition 1.11 to the resolution for $\mathcal{O}_{W}$ shows that $\mathcal{E} x t^{i}\left(\mathcal{E}^{\vee}, \mathcal{O}\right)=0$ for $0<i<r$.

Proceeding as suggested above, let $X$ be a complete intersection of $r$ hypersurfaces of degrees $d_{i}$ which links $V$ to another subscheme $W$. Let $F$ denote the Koszul complex for $\mathcal{O}_{X}$, so that $F_{0}=\mathcal{O}, F_{1}=\bigoplus \mathcal{O}\left(-d_{i}\right)$ and $F_{r}=\mathcal{O}\left(-\sum d_{i}\right)$. By looking at the maps involved, it's easy to see that the dual complex $F^{\vee}$ is a Koszul resolution for $\mathcal{O}_{X} \otimes F_{r}^{\vee}$. Let $\alpha$ be a morphism given by Proposition 1.8, which maps the Koszul resolution $F$ of $\mathcal{O}_{X}$ to the $\mathcal{E}$-type resolution of $\mathcal{O}_{V}$. Dualizing $\alpha$ and applying the mapping cone procedure (Proposition 1.8) gives a resolution for $\mathcal{O}_{W} \otimes F_{r}^{\vee}$. This complex, along with a morphism of the (dual) Koszul complex for $\mathcal{O}_{X} \otimes F_{r}^{\vee}$ to this complex, is given in the diagram below.

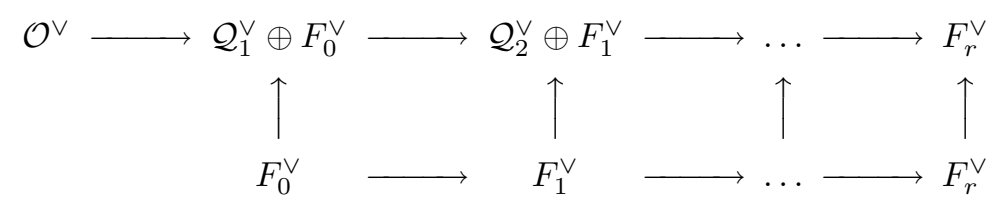

The differential on the mapping cone complex is given by the usual formula, namely, $\delta(x, y)=(\delta(x), \alpha(x)-\delta(y))$. Given that this is the differential, it is easy to see that the diagram commutes if we take the morphism of complexes to be the inclusion of the second factor. Further, we can cancel off the redundant copy of $\mathcal{O}^{\vee}$ and $F_{0}^{\vee}$ on the left if we replace the vertical map from $F_{0}^{\vee}$ to $\mathcal{Q}_{1}^{\vee} \oplus F_{0}^{\vee}$ with the map $F_{0}^{\vee}=\mathcal{O}^{\vee} \stackrel{\pi^{\vee}}{\longrightarrow} \mathcal{Q}_{1}^{\vee}$.

Using this morphism of complexes, we apply Proposition 1.11. We dualize the modified morphism of complexes. After removing all the double duals, we get a diagram

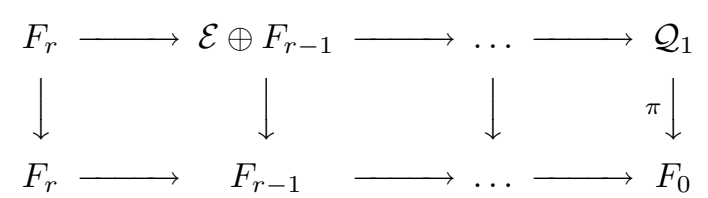

Here the leftmost vertical map is the identity, the middle vertical maps are projections onto the second factor, and the rightmost vertical map is $\pi$. After splitting off the extra summand of $F_{r}$ that appears, the mapping cone takes the form

$$
0 \rightarrow \mathcal{E} \oplus F_{r-1} \rightarrow \mathcal{Q}_{r-1} \oplus F_{r-2} \oplus F_{r-1} \rightarrow \ldots \rightarrow \mathcal{Q}_{1} \oplus F_{1} \rightarrow F_{0}
$$

The induced map on the summands $F_{i}$ is the identity, so these can also be removed. Looking at the induced maps, we see that this is the complex for $\mathcal{O}_{X}$ that we 
started with. Since $V$ is linked to $W$, we can apply Proposition 1.11 to see that $\mathcal{E} x t^{i}\left(\left(\mathcal{E}^{\vee} \oplus F_{r-1}^{\vee}\right) \otimes F_{r}, \mathcal{O}\right)=0$ for $0<i<r$. Since $F_{r-1}^{\vee}$ and $F_{r}$ are dissocié, we conclude that $\mathcal{E} x t^{i}\left(\mathcal{E}^{\vee}, \mathcal{O}\right)=0$ for $0<i<r$. This finishes the proof.

Remark 1.17. Put into the context of commutative algebra, the proof above yields the following result: Let $A$ be a regular local ring, $I \subset A$ an ideal such that all the associated primes to $A / I$ have height $r$. If

$$
0 \rightarrow E \rightarrow F_{r-1} \rightarrow F_{r-2} \rightarrow \ldots \rightarrow F_{0}(\rightarrow A / I \rightarrow 0)
$$

is a resolution of $A / I$ with $F_{i}$ free, then $\operatorname{Ext}^{i}\left(E^{\vee}, A\right)=0$ for each $0<i<r$.

Corollary 1.18. Let $V \subset \mathbb{P}^{n}$ be a nonempty subscheme of codimension $\geq r$. Then $V$ is of pure codimension $r$ if and only if for every $\mathcal{E}$-type resolution

$$
0 \rightarrow \mathcal{E} \rightarrow \mathcal{Q}_{r-1} \rightarrow \mathcal{Q}_{r-2} \rightarrow \ldots \rightarrow \mathcal{Q}_{1} \rightarrow \mathcal{O}\left(\rightarrow \mathcal{O}_{V} \rightarrow 0\right)
$$

we have that $\mathcal{E} x t^{i}\left(\mathcal{E}^{\vee}, \mathcal{O}\right)=0$ for $0<i<r$.

Proof. Proposition 1.16 gives us the forward direction. Conversely, suppose that $V$ has such a resolution. Since $V$ has codimension $\geq r$, we can find a complete intersection scheme $X$ which contains $V$. Letting $\alpha$ be a morphism from the Koszul resolution of $\mathcal{O}_{X}$ to the $\mathcal{E}$-type resolution for $X$, we apply Proposition 1.8 to see that the mapping cone of $\alpha^{\vee}(-d)$ gives a resolution for $\mathcal{O}_{W}$, where $W \subset X$ is defined by $\mathcal{I}_{W, X} \cong \mathcal{H o m}_{\mathcal{O}_{X}}\left(\mathcal{O}_{V}, \mathcal{O}_{X}\right)$. By our condition on $\mathcal{E}$, we see that this is an $\mathcal{N}$-type resolution for $W$.

Now we can apply Proposition 1.11 to the resolution for $W$. If we use the morphism of complexes chosen in the proof of Proposition 1.16, we get an $\mathcal{E}$-type resolution for a scheme $V^{\prime}$ defined by $\mathcal{I}_{V^{\prime}, X} \cong \mathcal{H o m}_{\mathcal{O}_{X}}\left(\mathcal{O}_{W}, \mathcal{O}_{X}\right)$. By choice of the morphism, we see that the resolution can be simplified to agree with our starting resolution for $V$. It follows that $V^{\prime}=V$ and that $V$ and $W$ are linked by the complete intersection $X$. In particular, $V$ is pure codimension $r$.

Remark 1.19. The corresponding commutative algebra statement is as follows: Let $(A, m)$ be a regular local ring, and let $I \subset A$ be an ideal such that all the associated primes of $A / I$ have height $\geq r$. If

$$
0 \rightarrow E \rightarrow F_{r-1} \rightarrow F_{r-2} \rightarrow \ldots \rightarrow F_{0}(\rightarrow A / I \rightarrow 0)
$$

is a resolution of $A / I$ with $F_{i}$ free, then all associated primes of $A / I$ have height exactly $r \Longleftrightarrow \operatorname{Ext}^{i}(\operatorname{Hom}(E, A), A)=0$ for $0<i<r$. This local version shows that the global version can be strengthened in the following way: if we only assume that the $\mathcal{Q}_{i}$ are locally free, then $V$ has pure codimension $r \Longleftrightarrow \mathcal{E} x t^{i}\left(\mathcal{E}^{\vee}, \mathcal{O}\right)=0$ for $0<i<r$.

Corollary 1.20. Let $V \subset \mathbb{P}^{n}$ be a nonempty subscheme of codimension $\geq r$. Then $V$ is of pure codimension $r$ if and only if $V$ has an $\mathcal{N}$-type resolution.

Proof. If $V$ is of pure codimension $r$, we can link $V$ to a subscheme $W$ of pure codimension $r$. If we use an $\mathcal{E}$-type resolution for $W$ and apply Propositions 1.16 and 1.11 , we get an $\mathcal{N}$-type resolution for $V$ as described in the corollary. 
Conversely, suppose that $V \subset \mathbb{P}^{n}$ has an $\mathcal{N}$-type resolution. Since $V$ has codimension $\geq r$, we can find a complete intersection $X$ which contains $V$. Let $\alpha$ be a morphism from the Koszul complex of $\mathcal{O}_{X}$ to the $\mathcal{N}$-type resolution of $V$. Applying Proposition 1.11, we see that the mapping cone of $\alpha^{\vee}(-d)$ gives an $\mathcal{E}$-type resolution for a subscheme $W \subset X$ defined by $\mathcal{I}_{W, X} \cong \mathcal{H o m}_{\mathcal{O}_{X}}\left(\mathcal{O}_{V}, \mathcal{O}_{X}\right)$. As in the proof of Proposition 1.16, we can find a morphism from the Koszul complex of $\mathcal{O}_{X}$ to this $\mathcal{E}$-type resolution in such a way that the cone of the dual morphism twisted by $-d$ simplifies to the original $\mathcal{N}$ type resolution for $V$. It follows (as in the previous corollary) that $V$ is linked to $W$, hence $V$ is of pure codimension $r$.

Remark 1.21. The reflexive sheaves $\mathcal{E}$ such that $\mathcal{E} x t^{i}\left(\mathcal{E}^{\vee}, \mathcal{O}\right)=0$ for $0<i<r$ give a nice generalization of vector bundle. In the context of Corollary 1.18, the sheaf $\mathcal{E}$ is locally free if and only if the scheme $V$ is locally Cohen-Macaulay. Dually, the reflexive sheaves $\mathcal{N}$ such that $\mathcal{E} x t^{i}(\mathcal{N}, \mathcal{O})=0$ for $0<i<r$ generalize the notion of a vector bundle. In the context of Corollary 1.20, we again have that $\mathcal{N}$ is locally free if and only if the subscheme $V$ is locally Cohen-Macaulay.

\section{RAO'S CORRESPONDENCE}

In this section, we extend Rao's correspondence between stable equivalence classes of vector bundles and even linkage classes of Cohen-Macaulay subschemes of codimension two. By specializing the results of the previous section to the case $r=2$, we find that we can remove the Cohen-Macaulay hypothesis on the even linkage classes if we use stable equivalence classes of reflexive sheaves $\mathcal{E}$ such that $H_{*}^{1}(\mathcal{E})=0$ and $\mathcal{E} x t^{1}\left(\mathcal{E}^{\vee}, \mathcal{O}\right)=0$. We follow Rao's proof [16] with few changes.

Definition 2.1. The equivalence relation generated by linking is called liaison or linkage. If two schemes can be connected by an even number of linkages, we say that they are evenly linked and the corresponding equivalence relation is called biliaison or even linkage.

Definition 2.2. Two reflexive sheaves $\mathcal{E}_{1}$ and $\mathcal{E}_{2}$ on $\mathbb{P}^{n}$ are stably equivalent if there exist dissocié sheaves $\mathcal{Q}_{1}, \mathcal{Q}_{2}$ and $h \in \mathbb{Z}$ such that $\mathcal{E}_{1} \oplus \mathcal{Q}_{1} \cong \mathcal{E}_{2}(h) \oplus \mathcal{Q}_{2}$. This is an equivalence relation among reflexive sheaves on $\mathbb{P}^{n}$.

Proposition 2.3. Let $\Omega$ be a stable equivalence class of reflexive sheaves on $\mathbb{P}^{n}$. Let $\mathcal{F}_{0}$ be a sheaf in $\Omega$ of minimal rank. Then for each $\mathcal{G} \in \Omega$, there exists $h \in \mathbb{Z}$ and a dissocié sheaf $\mathcal{Q}$ such that $\mathcal{G} \cong \mathcal{F}_{0}(h) \oplus \mathcal{Q}$. In particular, the isomorphism class of $\mathcal{F}_{0}$ is uniquely determined up to twist.

Proof. Let $\mathcal{F}_{0}$ be as in the statement, and let $\mathcal{G} \in \Omega$ be arbitrary. Since $\mathcal{F}_{0}$ and $\mathcal{G}$ are in the same stable equivalence class, there exists $h \in \mathbb{Z}$ and dissocie sheaves $\mathcal{Q}_{1}, \mathcal{Q}_{2}$ such that $\mathcal{F}_{0}(h) \oplus \mathcal{Q}_{1} \cong \mathcal{G} \oplus \mathcal{Q}_{2}$. Let $\mathcal{O}(-a)$ be a line bundle summand of $\mathcal{Q}_{2}$ and write $\mathcal{Q}_{2}=\mathcal{O}(-a) \oplus \mathcal{Q}_{2}^{\prime}$ with $\mathcal{Q}_{2}^{\prime}$ dissocié. If we twist the isomorphism by $a$ and consider the map on global sections, we obtain a surjection

$$
H^{0}\left(\mathcal{F}_{0}(h+a)\right) \oplus H^{0}\left(\mathcal{Q}_{1}(a)\right) \rightarrow H^{0}(\mathcal{O})=k
$$

If the induced map $H^{0}\left(\mathcal{F}_{0}(h+a)\right) \rightarrow k$ is surjective, then the corresponding map $\mathcal{F}_{0}(h+a) \rightarrow \mathcal{O}$ is a split surjection. If $\mathcal{E}$ is the kernel, then $\mathcal{E} \oplus \mathcal{O} \cong \mathcal{F}_{0}(h+a)$ shows that $\mathcal{F}_{0}$ was not of minimal rank in $\Omega$, a contradiction. It follows that the induced map is zero, and hence the map $H^{0}\left(\mathcal{Q}_{1}(a)\right) \rightarrow k$ is surjective. In this case 
the induced map $\mathcal{Q}_{1} \rightarrow \mathcal{O}(-a)$ is split surjective, and if $\mathcal{Q}_{1}^{\prime}$ is the kernel, then we can split off $\mathcal{O}(-a)$ from the original isomorphism to get

$$
\mathcal{F}_{0}(h) \oplus \mathcal{Q}_{1}^{\prime} \cong \mathcal{G} \oplus \mathcal{Q}_{2}^{\prime}
$$

Continuing in this fashion, we can split off all the summands of $\mathcal{Q}_{2}$ to obtain an isomorphism $\mathcal{G} \cong \mathcal{F}_{0}(h) \oplus \mathcal{Q}$ as in the proposition.

Proposition 2.4. There is a well-defined map $\Phi$ from even linkage classes of pure codimension two subschemes of $\mathbb{P}^{n}$ to stable equivalence classes of reflexive sheaves $\mathcal{E}$ such that $H_{*}^{1}(\mathcal{E})=0$ and $\mathcal{E} x t^{1}\left(\mathcal{E}^{\vee}, \mathcal{O}\right)=0$. This map is given by taking $X$ to the last sheaf in an $\mathcal{E}$-type resolution for $X$.

Proof. The map has been given above. It is clear from the definition of $\mathcal{E}$-type resolution and Proposition 1.16 that the image of $\Phi$ consists of reflexive sheaves $\mathcal{E}$ satisfying $H_{*}^{1}(\mathcal{E})=0$ and $\mathcal{E} x t^{1}\left(\mathcal{E}^{\vee}, \mathcal{O}\right)=0$. We need only show that it is welldefined. For this, assume that $X_{1}, X_{2}$ are in the same even linkage class and let

$$
0 \rightarrow \mathcal{E}_{1} \rightarrow \mathcal{Q}_{1} \rightarrow \mathcal{O}\left(\rightarrow \mathcal{O}_{X_{1}} \rightarrow 0\right)
$$

be an $\mathcal{E}$-type resolution for $X_{1}$. By alternately applying Propositions 1.8 and 1.11 we arrive at a resolution for $X_{2}$ of the form

$$
0 \rightarrow \mathcal{E}_{1}(h) \oplus \mathcal{P}_{2} \rightarrow \mathcal{Q}_{2} \rightarrow \mathcal{O}\left(\rightarrow \mathcal{O}_{X_{2}} \rightarrow 0\right)
$$

where $\mathcal{P}_{2}$ and $\mathcal{Q}_{2}$ are dissocié. Clearly $\mathcal{E}_{1}$ and $\mathcal{E}_{1}(h) \oplus \mathcal{P}_{2}$ are in the same stable equivalence class, so it suffices to show that if $X_{2}$ has a second $\mathcal{E}$-type resolution

$$
0 \rightarrow \mathcal{F} \rightarrow \mathcal{Q} \rightarrow \mathcal{I}_{X_{2}} \rightarrow 0
$$

then $\mathcal{F}$ is stably equivalent to $\mathcal{E}_{1}(h) \oplus \mathcal{P}_{2}$.

For this, we add the map $\mathcal{Q}_{2} \rightarrow \mathcal{I}_{X_{2}}$ to the resolution involving $\mathcal{F}$, to obtain a commutative diagram

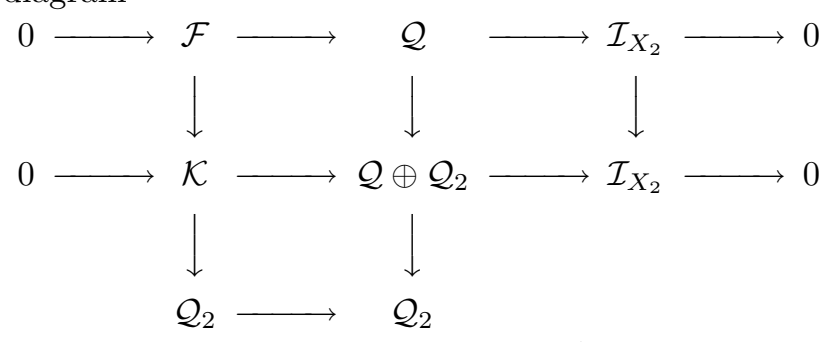

where the columns and rows are exact. Since $H_{*}^{1}(\mathcal{F})=0$ by assumption, the leftmost column shows that $H_{*}^{0}(\mathcal{K}) \rightarrow H_{*}^{0}\left(\mathcal{Q}_{2}\right)$ is surjective. Since $\mathcal{Q}_{2}$ is dissocié, it follows that the map $\mathcal{K} \rightarrow \mathcal{Q}_{2}$ is split surjective, whence $\mathcal{K} \cong \mathcal{F} \oplus \mathcal{Q}_{2}$. Applying the same argument to the other resolution of $\mathcal{I}_{X_{2}}$ shows that $\mathcal{K} \cong \mathcal{E}_{1}(h) \oplus \mathcal{P}_{2} \oplus \mathcal{Q}$, so that

$$
\mathcal{F} \oplus \mathcal{Q}_{2} \cong \mathcal{K} \cong \mathcal{E}_{1}(h) \oplus \mathcal{P}_{2} \oplus \mathcal{Q}
$$

which shows that $\mathcal{F}$ is stably equivalent to $\mathcal{E}_{1}$.

Lemma 2.5. Let $X \subset \mathbb{P}^{n}$ be a subscheme of pure codimension two having an $\mathcal{N}$-type resolution

$$
0 \rightarrow \mathcal{P} \rightarrow \mathcal{N} \stackrel{\pi}{\longrightarrow} \mathcal{I}_{X} \rightarrow 0
$$

If $\mathcal{N} \cong \mathcal{N}^{\prime} \oplus \mathcal{O}(-p)$, then there exists a subscheme $X^{\prime}$ in the even linkage class of $X$ which has a an $\mathcal{N}$-type resolution of the form

$$
0 \rightarrow \mathcal{P}^{\prime} \rightarrow \mathcal{N}^{\prime}(d) \rightarrow \mathcal{I}_{X^{\prime}} \rightarrow 0
$$

for some $d \in \mathbb{Z}$. 
Proof. First note that $\mathcal{E} x t^{1}\left(\mathcal{N}^{\prime}, \mathcal{O}\right)=0$, because $\mathcal{E} x t$ respects direct sums. Moreover, the fact that dualizing respects direct sums shows that $\mathcal{N}^{\prime}$ is reflexive and $H_{*}^{1}\left(\mathcal{N}^{\prime \vee}\right)=0$. These two facts show that it is at least possible to have an $\mathcal{N}$-type resolution involving $\mathcal{N}^{\prime}$.

Let $s \in H^{0}(\mathcal{N}(p))$ be a nowhere vanishing section corresponding to the summand $\mathcal{O}(-p)$ of $\mathcal{N}$ and let $\sigma: \mathcal{N}(p) \rightarrow \mathcal{O}$ be a fixed splitting of $s$ (the quotient of $\mathcal{N}(p)$ by $s$ is $\left.\mathcal{N}^{\prime}(p)\right)$. Let $f$ be the image of $s$ in $I_{X}$. As in [15, Proposition 1.11], we consider two cases.

Case 1: $f=0$. Let $\pi^{\prime}: \mathcal{N}^{\prime} \rightarrow \mathcal{I}_{X}$ be the composition of $\pi$ with the inclusion into $\mathcal{N}$, and let $\mathcal{P}^{\prime}$ be the kernel. The snake lemma gives the commutative diagram of short exact sequences below.

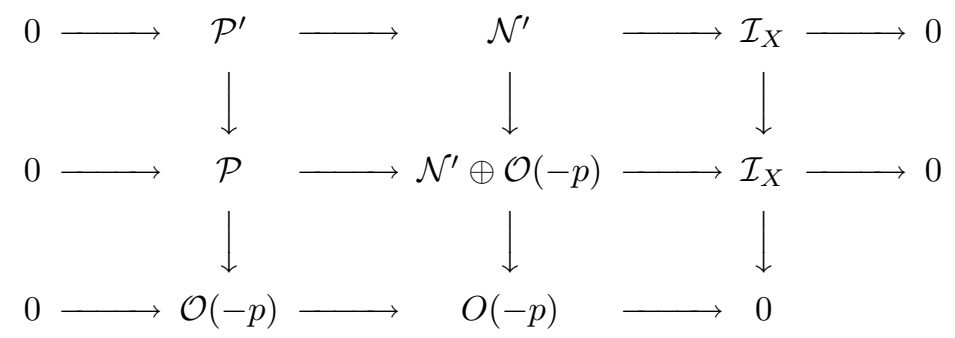

Since the map of graded modules $H_{*}^{0}\left(\mathcal{N}^{\prime}\right) \oplus H_{*}^{0}(\mathcal{O}(-p)) \rightarrow I_{X}$ is surjective and the induced map $H_{*}^{0}(\mathcal{O}(-p)) \rightarrow I_{X}$ is zero, the induced map $H_{*}^{0}\left(\mathcal{N}^{\prime}\right) \rightarrow I_{X}$ is also surjective. Since $H_{*}^{1}\left(\mathcal{N}^{\prime}\right) \cong H_{*}^{1}\left(\mathcal{I}_{X}\right)$ via $\pi^{\prime}$, we see that $H_{*}^{1}\left(\mathcal{P}^{\prime}\right)=0$. Since $\mathcal{P}$ is dissocié, the leftmost column shows that $H_{*}^{i}\left(\mathcal{P}^{\prime}\right)=0$ for $2 \leq i<n$. Combining with the previous observation, we see that all the middle cohomology of $\mathcal{P}^{\prime}$ vanishes. Applying [4, Lemma 6.3], it follows that $\mathcal{P}^{\prime}$ is dissocié. This finishes the case $f=0$.

Case 2: $f \neq 0$. In this case $f$ gives the equation of a hypersurface $S$ of degree $p$ which contains $X$. Let $T$ be another hypersurface (of degree $t$ ) which meets $S$ properly. If we use this complete intersection to link $X$ to $W$, then by Proposition 1.11 we have an exact sequence

$$
0 \rightarrow \mathcal{N}^{\vee}(-p-t) \rightarrow \mathcal{P}^{\vee}(-p-t) \oplus \mathcal{O}(-p) \oplus \mathcal{O}(-t) \rightarrow \mathcal{I}_{W} \rightarrow 0
$$

where the composition of the first map and projection to $\mathcal{O}(-t)$ is precisely the map $\mathcal{N}^{\vee} \rightarrow \mathcal{O}(p)$ tensored by $\mathcal{O}(-s-t)$, which is split surjective. Now we can form a surjective morphism of exact sequences

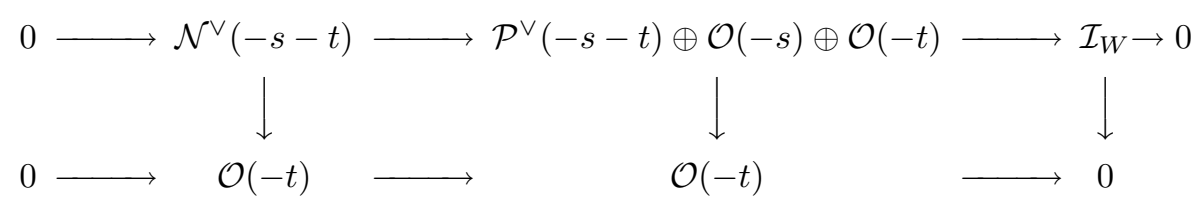

Applying the snake lemma gives an exact sequence of kernels

$$
0 \rightarrow \mathcal{N}^{\prime \vee}(-p-t) \rightarrow \mathcal{P}^{\vee}(-p-t) \oplus \mathcal{O}(-p) \rightarrow \mathcal{I}_{W} \rightarrow 0
$$

We can choose a pair of equations in $I_{W}$ which are images of generators of two of the line bundle summands in $\mathcal{P}^{\vee}(-p-t) \oplus \mathcal{O}(-p)$ and give hypersurfaces $S^{\prime}, T^{\prime}$ of 
degrees $s^{\prime}, t^{\prime}$ which meet properly. Applying Proposition 1.8, we get a resolution for the subscheme $X^{\prime}$ linked to $W$ by $S^{\prime} \cap T^{\prime}$. Because of our choices of hypersurface, we can split off the two line bundle summands corresponding to the equations of $S^{\prime}$ and $T^{\prime}$, which leaves an $\mathcal{N}$-type resolution for $X^{\prime}$ with just $\mathcal{N}^{\prime}\left(p+t-s^{\prime}-t^{\prime}\right)$ in the middle.

Remark 2.6. Comparing with [15, Proposition 1.11], we see that the statement here is somewhat weaker, as the section of $\mathcal{N}$ considered is split instead of merely nowhere vanishing. The reason is that the case $f=0$ in Rao's proof is flawed, but weakening the hypotheses allows the proof to be repaired. Fortunately, the weaker statement is still enough to prove Proposition 2.8, so Rao's theorem is not compromised.

Lemma 2.7. Let $\mathcal{N}$ be a reflexive sheaf such that $H_{*}^{1}\left(\mathcal{N}^{\vee}\right)=0$ and $\mathcal{E} x t^{1}(\mathcal{N}, \mathcal{O})=$ 0 . Let $U \subset \mathbb{P}^{n}$ be an open subscheme whose complement has codimension $\geq 2$. If $Y \subset U$ is a closed subscheme of codimension two (or empty) which has a resolution of the form

$$
0 \rightarrow \mathcal{P}_{U} \rightarrow \mathcal{N}_{U} \rightarrow \mathcal{I}_{Y, U}(t) \rightarrow 0
$$

with $\mathcal{P}$ dissocié, then there exists a subscheme $\bar{Y} \subset \mathbb{P}^{n}$ of pure codimension two which agrees with $Y$ on $U$ and has a resolution

$$
0 \rightarrow \mathcal{P} \rightarrow \mathcal{N} \rightarrow \mathcal{I}_{\bar{Y}}(t) \rightarrow 0
$$

In particular, if the codimension of the complement of $U$ is $\geq 3$, then $\bar{Y}$ is the scheme-theoretic closure of $Y$ in $\mathbb{P}^{n}$.

Proof. Let $j: U \rightarrow \mathbb{P}^{n}$ be the inclusion. Since both $\mathcal{P}$ and $\mathcal{N}$ are reflexive, we see from $\left[6\right.$, Proposition 2.5] that $j_{*}\left(\mathcal{P}_{U}\right)=\mathcal{P}$ and $j_{*}\left(\mathcal{N}_{U}\right)=\mathcal{N}$. The left exactness of $j_{*}$ gives an exact sequence

$$
0 \rightarrow \mathcal{P} \rightarrow \mathcal{N} \rightarrow j_{*}\left(\mathcal{I}_{Y, U}(t)\right)
$$

The left exactness of $j_{*}$ shows that $j_{*}\left(\mathcal{I}_{Y, U}(t)\right)$ is a subsheaf of $\mathcal{O}(t)$, so we get an exact sequence

$$
0 \rightarrow \mathcal{P} \rightarrow \mathcal{N} \rightarrow \mathcal{I}_{\bar{Y}}(t) \rightarrow 0
$$

where $\bar{Y}$ is a closed subscheme which agrees with $Y$ on $U$. By Corollary 1.18, this exact sequence shows that $\bar{Y}$ is of pure codimension two or empty, and hence the same goes for $Y$. If the complement of $U$ has codimension $\geq 3$, then $\bar{Y}$ can only be the scheme-theoretic closure of $Y$ (because $U$ must contain all the generic points of each).

Proposition 2.8. Let $X_{1}, X_{2} \subset \mathbb{P}^{n}$ be two subschemes of pure codimension two having $\mathcal{N}$-type resolutions of the form

$$
\begin{aligned}
& 0 \rightarrow \bigoplus_{j=1}^{r} \mathcal{O}\left(a_{j}\right) \stackrel{\alpha}{\rightarrow} \mathcal{F}(a) \rightarrow \mathcal{I}_{X_{1}} \rightarrow 0 \\
& 0 \rightarrow \bigoplus_{j=1}^{r} \mathcal{O}\left(b_{j}\right) \stackrel{\beta}{\rightarrow} \mathcal{F}(b) \rightarrow \mathcal{I}_{X_{2}} \rightarrow 0 .
\end{aligned}
$$


Then $X_{1}, X_{2}$ belong to the same even linkage class.

Proof. If $\mathcal{F}$ is dissocié, then both $X_{1}$ and $X_{2}$ are in the linkage class of Arithmetically Cohen-Macaulay subschemes. This linkage class is in fact an even linkage class, because it contains the complete interection of two hyperplanes, which is self-linked.

We now assume that $\mathcal{F}$ is not dissocié. In this case, Proposition 2.3 shows that we can split off line bundles from $\mathcal{F}$ until we obtain a representative $\mathcal{F}_{0}$ for the stable equivalence class for $\mathcal{F}$ of minimal degree. Applying Lemma 2.5 repeatedly allows us to replace $X_{1}, X_{2}$ with representatives from their respective even linkage classes which have resolutions as above involving $\mathcal{F}_{0}$. Thus we reduce to the case where $\mathcal{F}$ has no line bundle summands.

Now the proof is identical to Rao's proof (with one slight change) of the corresponding lemma for locally free sheaves [15, Lemma 1.6], which we repeat for the reader's convenience. The maps $\alpha$ and $\beta$ are described by two sequences $\left\{s_{i}\right\}$ and $\left\{t_{i}\right\}$ of global sections of various twists of $\mathcal{F}$. We will assume that $s_{i}=t_{i}$ for $i \leq j<r$ and show how to replace $X_{1}, X_{2}$ with subschemes with resolutions in which we have $s_{j+1}=t_{j+1}$.

For this, we can find general sections $r_{1}, r_{2}$ of $\mathcal{F}(p)$ for $p>>0$ whose images in $I_{X_{1}}$ give rise to independent hypersurfaces $S_{11}$ and $S_{12}$ containing $X_{1}$ and whose images in $I_{X_{2}}$ give rise to independent hypersurfaces $S_{21}$ and $S_{22}$ containing $X_{2}$. Using these hypersurfaces, we link $X_{1}$ to $Y_{1}$ and $X_{2}$ to $Y_{2}$. By Proposition 1.11, $Y_{1}$ has an $\mathcal{E}$-type resolution

$$
0 \rightarrow \mathcal{F}^{\vee}(a-2 p) \rightarrow \bigoplus_{i=1}^{r} \mathcal{O}\left(2 a-2 p-a_{i}\right) \oplus \mathcal{O}(a-p)^{2} \rightarrow \mathcal{I}_{Y_{2}} \rightarrow 0
$$

where the restriction of the first map to the summand $\bigoplus \mathcal{O}\left(2 a-2 p-a_{i}\right)$ is the dual map to $\alpha$. By our initial reduction, none of the sections $s_{i}$ of $\mathcal{F}$ can be split off. Consider the induced map $\mathcal{O}\left(2 a-2 p-a_{j+1}\right) \rightarrow \mathcal{I}_{Y_{1}}$. If this map is zero, then the map induced map $H^{0}(\mathcal{O}) \rightarrow H^{0}\left(\mathcal{I}_{Y_{1}}\left(-2 a+2 p+a_{j+1}\right)\right)$ is clearly zero. Twisting the sequence above by $-2 a+2 p+a_{j+1}$, we see that the map $H^{0}\left(\mathcal{F}^{\vee}\left(-a+a_{j+1}\right)\right) \rightarrow$ $H^{0}(\mathcal{O})$ is surjective, and we deduce that the induced map $\mathcal{F}^{\vee}\left(-a+a_{j+1}\right) \rightarrow \mathcal{O}$ is a split surjection. It follows that the section $\mathcal{O}\left(-a_{j+1}\right) \rightarrow \mathcal{F}(a)$ is also split, which contradicts our original reduction.

It follows from the above paragraph that the map $\mathcal{O}\left(2 a-2 p-a_{j+1}\right) \rightarrow \mathcal{I}_{Y_{1}}$ is nonzero and hence defines a hypersurface $T_{1}$ containing $Y_{1}$. Similarly the image of $\mathcal{O}\left(2 b-2 p-b_{j+1}\right)$ in $\mathcal{I}_{Y_{2}}$ gives a hypersurface $T_{2}$ which contains $Y_{2}$. If we replace the sections $r_{1}, r_{2}$ with a general linear combination $l r_{1}+m r_{2}, h r_{1}+k r_{2}$, we will get new pairs of hypersurfaces containing $X_{1}, X_{2}$ which form the same complete intersections $S_{11} \cap S_{12}$ and $S_{21} \cap S_{22}$. If we replace $r_{1}, r_{2}$ with a sufficiently general linear combination, the corresponding hypersurface $S_{11}$ (respectively $S_{21}$ ) will meet $T_{1}$ (respectively $T_{2}$ ) properly. We use the corresponding complete intersection $S_{11} \cap$ $T_{1}$ (respectively $S_{21} \cap T_{2}$ ) to link $Y_{1}$ (respectively $Y_{2}$ ) to $X_{1}^{1}$ (respectively $X_{2}^{1}$ ).

By Corollary 1.10, we obtain a resolution

$0 \rightarrow \bigoplus_{i \neq j+1} \mathcal{O}\left(a_{i}-a_{j+1}+a-p\right) \oplus \mathcal{O}\left(2 a-2 p-a_{j+1}\right) \stackrel{\alpha^{1}}{\longrightarrow} \mathcal{F}\left(2 a-p-a_{j+1}\right) \rightarrow \mathcal{I}_{X_{1}^{1}} \rightarrow 0$ 
where the map $\alpha^{1}$ is given by the sequence

$$
\left\{s_{1}, \ldots, s_{j}, s_{j+2}, \ldots, s_{r},-r_{1}\right\}
$$

and we have effectively replaced $s_{j+1}$ with $r_{1}$. The analogous statement holds for the resolution of $X_{2}^{1}$. We repeat this process until we obtain subschemes $X_{1}^{*}, X_{2}^{*}$ in the respective even linkage classes of $X_{1}, X_{2}$ such that the there are $\mathcal{N}$-type resolutions with maps $\alpha^{*}, \beta^{*}$ which give the same sections of $\mathcal{F}$. On the open set $U$ where $\mathcal{F}$ is locally free, we see that $X_{1}^{*}=X_{2}^{*}$ because the ideals of both are given by the maximal minors of $\alpha^{*}=\beta^{*}$. Now apply Lemma 2.7 to see that $X_{1}^{*}=X_{2}^{*}$. This shows that $X_{1}$ is evenly linked to $X_{2}$, proving the proposition.

Corollary 2.9. The map $\Phi$ from Proposition 2.4 is injective.

Proof. Suppose that two schemes $Y_{1}, Y_{2}$ have $\mathcal{E}$-type resolutions

$$
0 \rightarrow \mathcal{E}_{i} \rightarrow \mathcal{Q}_{i} \rightarrow \mathcal{I}_{Y_{i}} \rightarrow 0
$$

where $\mathcal{E}_{1}$ is stably equivalent to $\mathcal{E}_{2}$. By adding some extra summands we may assume that $\mathcal{E}_{1} \cong \mathcal{E}_{2}(d)$ for some $d \in \mathbb{Z}$. If we choose two equations in each of the ideals $I_{Y_{i}}$ which come from generators for line bundle summands of $\mathcal{Q}_{i}$ and define hypersurfaces which meet properly, then we can link $Y_{1}, Y_{2}$ via these hypersurfaces to $X_{1}, X_{2}$. Using Proposition 1.8 we get $\mathcal{N}$-type resolutions for the $X_{i}$, and by our choice of hypersurfaces, we can split off the pair of summands corresponding to the hypersurfaces used for the linkage. In this case, we get $\mathcal{N}$-type resolutions as in the Lemma 2.8, and hence we can conclude that $X_{1}$ and $X_{2}$ are in the same even linkage class. It follows that $Y_{1}$ and $Y_{2}$ are also evenly linked.

Proposition 2.10. The map $\Phi$ from Proposition 2.4 is surjective.

Proof. Once again we follow Rao's proof. Let $\mathcal{E}$ be a reflexive sheaf such that $H_{*}^{1}(\mathcal{E})=0$ and $\mathcal{E} x t^{1}\left(\mathcal{E}^{\vee}, \mathcal{O}\right)=0$, and let $\mathcal{N}=\mathcal{E}^{\vee}$. The set where $\mathcal{N}$ fails to be locally free is closed of codimension $\geq 3$. Let $U$ be the open complement of this set with inclusion map $j: U \hookrightarrow \mathbb{P}^{n}$.

If we choose $p$ large enough that $\mathcal{N}(p)$ is generated by global sections, then $\mathcal{N}(p)_{U}$ is still generated by the same global sections. By quotienting out by nowhere vanishing sections, we obtain a quotient bundle $\mathcal{M}$ of rank $r \leq n$ which is still generated by global sections. Now we can apply [8, Theorem 3.3] to see that a general set of $r-1$ sections $\left\{s_{i}\right\}_{i=1}^{r-1}$ of $\mathcal{M}$ will have a degeneracy locus $Y$ of pure codimension two (on $U$ ). The generators of $\mathcal{I}_{Y}$ are locally given by the image of $\Lambda^{r-1} \gamma$, where $\gamma: \mathcal{O}^{r-1} \rightarrow \mathcal{M}$ is the map determined by the sections $s_{i}$. It follows that $Y$ is locally Cohen-Macaulay with resolution

$$
0 \rightarrow \mathcal{O}_{U}^{r-1} \rightarrow \mathcal{M} \rightarrow \mathcal{I}_{Y, U}(t) \rightarrow 0
$$

for some twist $t$. Since the kernel of the map $\mathcal{N}(p)_{U} \rightarrow \mathcal{M}$ was $\mathcal{O}_{U}^{l}$, where $l$ is the difference in rank between $\mathcal{N}$ and $\mathcal{M}$, we obtain an exact sequence

$$
0 \rightarrow \mathcal{O}_{U}^{r-1+l} \rightarrow \mathcal{N}(p)_{U} \rightarrow \mathcal{I}_{Y, U} \rightarrow 0
$$

Now we apply Lemma 2.7 to obtain an exact sequence

$$
0 \rightarrow \mathcal{O}^{r-1+l} \rightarrow \mathcal{N} \rightarrow \mathcal{I}_{\bar{Y}} \rightarrow 0
$$

where $\bar{Y}$ is of pure codimension two and in fact the scheme-theoretic closure of $Y$. If we link $\bar{Y}$ to another subscheme $X$, Proposition 1.11 shows that $X$ has an $\mathcal{E}$-type resolution with last step a twist of $\mathcal{E}$. This shows that $\Phi$ is surjective. 
Theorem 2.11. The even linkage classes of codimension two subschemes in $\mathbb{P}^{n}$ are in bijective correspondence with the stable equivalence classes of reflexive sheaves $\mathcal{E}$ on $\mathbb{P}^{n}$ satisfying $H_{*}^{1}(\mathcal{E})=0$ and $\mathcal{E} x t^{1}\left(\mathcal{E}^{\vee}, \mathcal{O}\right)=0$ via $\mathcal{E}$-type resolutions.

Theorem 2.12. The even linkage classes of codimension two subschemes in $\mathbb{P}^{n}$ are in bijective correspondence with the stable equivalence classes of reflexive sheaves $\mathcal{N}$ on $\mathbb{P}^{n}$ satisfying $H_{*}^{1}\left(\mathcal{N}^{\vee}\right)=0$ and $\mathcal{E} x t^{1}(\mathcal{N}, \mathcal{O})=0$ via $\mathcal{N}$-type resolutions.

Proof. This is evident from applying the mapping cone procedure to $\mathcal{E}$-type resolutions in the dual class.

Remark 2.13. In view of Proposition 2.3, the stable equivalence classes of reflexive sheaves are classified (up to twist) by certain reflexive sheaves which have no line bundle summands. If we think of stable equivalence classes as being determined by a sheaf of minimal rank, we see that via $\mathcal{E}$-type resolutions, the even linkage classes of codimension two subschemes are in bijective correspondence with isomorphism classes of reflexive sheaves $\mathcal{E}_{0}$ having no line bundle summands which satisfy $H_{*}^{1}\left(\mathcal{E}_{0}\right)=0$ and $\mathcal{E} x t^{1}\left(\mathcal{E}_{0}^{\vee}, \mathcal{O}\right)=0$, up to twist. In this case we will say that the even linkage class $\mathcal{L}$ corresponds to the stable equivalence class [ $\left.\mathcal{E}_{0}\right]$ via $\mathcal{E}$-type resolutions. Similarly, the even linkage classes of codimension two subschemes are in bijective correspondence with the isomorphism classes of reflexive sheaves $\mathcal{N}_{0}$ having no line bundle summands which satisfy $H_{*}^{1}\left(\mathcal{N}_{0}^{\vee}\right)=0$ and $\mathcal{E} x t^{1}\left(\mathcal{N}_{0}, \mathcal{O}\right)=0$, up to twist. In this case we say that the even linkage class $\mathcal{L}$ corresponds to the stable equivalence class $\left[\mathcal{N}_{0}\right]$ via $\mathcal{N}$-type resolutions.

Remark 2.14. A little checking shows that Proposition 2.4 generalizes to codimension $r$. In other words, by using the $\mathcal{E}$ in the $\mathcal{E}$-type resolution, we get a well-defined map from even linkage classes to stable equivalence classes of $r$ th syzygy modules. This map is not a bijection in the case when $r=n$, so Rao's correspondence does not extend to higher codimension without adaption.

\section{The Lazarsfeld-Rao Property}

Definition 3.1. Let $X \subset \mathbb{P}^{n}$ be a subscheme of pure codimension two. Let $S$ be a hypersurface of degree $s$ which contains $X$ and let $h \geq 0$ be an integer. We say that $Y$ is obtained from $X$ by a basic double link of height $h$ on $S$ if there is a hypersurface $H$ of degree $h$ and a hypersurface $T$ such that $S \cap T \operatorname{links} X$ to a subscheme $Z$, which in turn is linked to $Y$ by $S \cap(T \cup H)$.

Definition 3.2. Let $X, X^{\prime}$ be subschemes of pure codimension two in $\mathbb{P}^{n}$. We say that $X^{\prime}$ dominates $X$ at height $h \geq 0$ if $X^{\prime}$ can be obtained from $X$ by a sequence of basic double links with heights summing to $h$, followed by a deformation which preserves cohomology and even linkage class. In this case we write $X \leq_{h} X^{\prime}$, or simply $X \leq X^{\prime}$ if $h$ is not specified.

We will show that domination is transitive and hence defines a partial ordering on an even linkage class. Moreover, we will characterize domination in terms of the $\mathcal{N}$-type resolutions discussed in section 2 .

Lemma 3.3. Let $\mathcal{E} \stackrel{\phi}{\longrightarrow} \mathcal{Q}$ be an injective morphism of reflexive sheaves of the same degree on $\mathbb{P}^{n}$, where $\mathcal{E}$ has rank $r$ and $\mathcal{Q}$ has rank $r+1$. Assume that $\mathcal{E}$ and $\mathcal{Q}$ satisfy one of the following sets of conditions:

(A) $H_{*}^{1}(\mathcal{E})=0, \mathcal{E} x t^{1}\left(\mathcal{E}^{\vee}, \mathcal{O}\right)=0$ and $\mathcal{Q}$ is dissocié. 
(B) $H_{*}^{1}\left(\mathcal{Q}^{\vee}\right)=0, \mathcal{E} x t^{1}(\mathcal{Q}, \mathcal{O})=0$ and $\mathcal{E}$ is dissocié.

Then the following are equivalent.

1. The cokernel of $\phi$ is isomorphic to the ideal sheaf of a subscheme of pure codimension two.

2. The cokernel of $\phi$ is torsion free.

3. The complex

$$
0 \rightarrow \mathcal{E} \stackrel{\phi}{\longrightarrow} \mathcal{Q} \stackrel{\Lambda^{r} \phi^{\vee} \otimes 1}{\longrightarrow} \mathcal{O}
$$

is exact.

Proof. This is a generalized version of [10, II, Proposition 1.1]. We begin with a word of explanation about the complex of condition (3). We have an isomorphism $\mathcal{Q} \cong\left(\Lambda^{r} \mathcal{Q}\right)^{\vee} \otimes \Lambda^{r+1} \mathcal{Q}$ because these are isomorphic on the open set $U$ whose complement has codimension $\geq 2$ and both sides are reflexive. Since both $\mathcal{Q}$ and $\mathcal{E}$ have the same degree, we have that $\left(\Lambda^{r} \mathcal{E}\right)^{\vee} \otimes \Lambda^{r+1} \mathcal{Q} \cong \mathcal{O}$, which explains the map $\left(\Lambda^{r} \phi\right)^{\vee} \otimes 1$. To see that the sequence of condition (3) is a complex, first note that this holds on the open set $U$. If $j: U \hookrightarrow \mathbb{P}^{n}$ is the inclusion map, then applying $j_{*}$ to the sequence on $U$ gives the sequence from condition (3) because all three sheaves involved are reflexive. Since $j_{*}$ is a functor, the resulting sequence is a complex.

Now we may write the image of $\left(\Lambda^{r} \phi\right)^{\vee} \otimes 1$ as $\mathcal{I}(-\alpha)$, where $\mathcal{I}$ is the ideal sheaf of a subscheme in $\mathbb{P}^{n}$ of codimension $\geq 2$ and $\alpha \geq 0$. Letting $\mathcal{E}^{\prime}$ be the kernel of the induced map $\mathcal{Q} \rightarrow \mathcal{I}(-\alpha)$, we have a commutative diagram

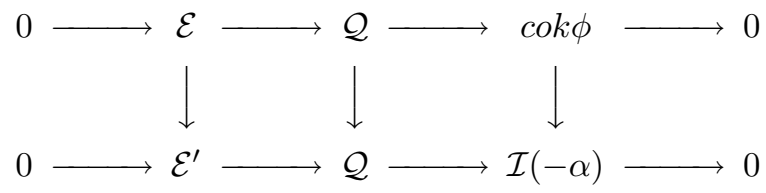

where the rows are short exact sequences. Clearly the cokernel of the inclusion $\mathcal{E} \rightarrow \mathcal{E}^{\prime}$ is a torsion sheaf, which is isomorphic to the kernel of the surjection $\operatorname{cok} \phi \rightarrow$ $\mathcal{I}(-\alpha)$ by the snake lemma. Now we show the equivalence of the conditions.

$(1) \Rightarrow(2)$ Any subsheaf of $\mathcal{O}_{\mathbb{P}^{n}}$ is torsion free.

$(2) \Rightarrow(3)$ Since the cokernel of $\phi$ is torsion free, the torsion sheaf $\mathcal{E}^{\prime} / \mathcal{E}$ must be zero, hence the complex of (3) is exact.

$(3) \Rightarrow(1)$ If the complex is exact, then the cokernel of $\phi$ is an ideal sheaf. The associated short exact sequence shows that the degree of this ideal sheaf is zero, hence the subscheme $Z$ that it defines is of codimension $\geq 2$. Now in either of the cases (A) or (B) given above, we can apply either Corollary 1.18 or Corollary 1.20 to see that $Z$ is of pure codimension two.

Lemma 3.4. Let $X \stackrel{f}{\rightarrow} Y$ be a morphism of schemes, and let $0 \rightarrow A \rightarrow B \rightarrow C \rightarrow 0$ be a complex of sheaves on $X$. Assume further that both $B$ and $C$ are flat over $Y$. If $y=f(x)$, then the set

$$
\left\{x \in X: 0 \rightarrow A_{x} \otimes k(y) \rightarrow B_{x} \otimes k(y) \rightarrow C_{x} \otimes k(y) \text { is exact }\right\}
$$

is open in $X$.

Proof. Let $I$ and $J$ denote the image and cokernel of the map $A \rightarrow B$. Since the sequence of the lemma is a complex, it can be factored as in the following 
commutative diagram:

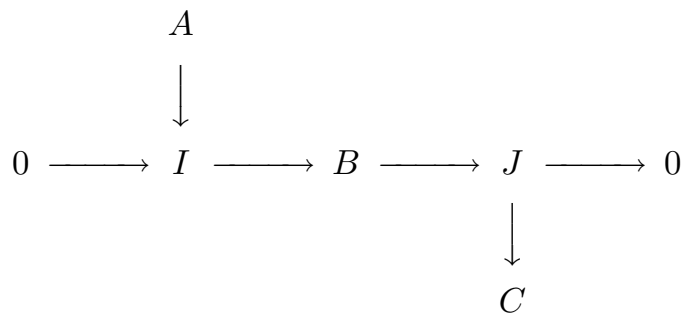

This diagram remains exact when we localize at $x$. Applying the right exact functor $\otimes k(y)$, we see that the sequence

$$
0 \rightarrow A_{x} \otimes k(y) \rightarrow B_{x} \otimes k(y) \rightarrow C_{x} \otimes k(y)
$$

is exact if and only if the maps $A_{x} \otimes k(y) \rightarrow B_{x} \otimes k(y)$ and $J_{x} \otimes k(y) \rightarrow C_{x} \otimes k(y)$ are injective. Since $B$ and $C$ are both flat over $Y$, the set of $x \in X$ such that both these maps are injective is open by [3, IV, Corollary 11.3].

Corollary 3.5. Let $X \stackrel{f}{\rightarrow} Y$ be a proper flat morphism of noetherian schemes, and let $0 \rightarrow A \rightarrow B \rightarrow C \rightarrow 0$ be a complex on $X$ with $B$ and $C$ locally free. Then the set

$$
U=\{y \in Y: 0 \rightarrow A \otimes k(y) \rightarrow B \otimes k(y) \rightarrow C \otimes k(y) \text { is exact }\}
$$

is open in $Y$.

Proof. Let $y \in Y$. Then $y \in U$ if and only if the sequence $0 \rightarrow A_{x} \otimes k(y) \rightarrow$ $B_{x} \otimes k(y) \rightarrow C_{x} \otimes k(y)$ is exact for each $x \in f^{-1}(y)$. This condition is open on $X$ by the previous lemma, because both $B$ and $C$ are flat over $Y$ in this case. If $y \in U$, then we can find an open set $V_{x}$ for each $x \in f^{-1}(y)$ giving the exactness condition. Since $f^{-1}(y)$ is noetherian, a finite number of these open sets cover $f^{-1}(y)$, call them $\left\{V_{i}\right\}_{1}^{n}$. Since $f$ is proper, the set $f\left(X-\cup V_{i}\right)$ is closed in $Y$ and avoids $y$. Hence there is an open neighborhood $W$ of $y$ which also avoids the set $f\left(X-\cup V_{i}\right)$. Since for any $w \in W$ we have that $f^{-1}(w) \subset \cup V_{i}$, we see that $W \subset U$. This shows that $U$ is open.

Lemma 3.6. Let $\mathcal{L}$ be an even linkage class of subschemes of pure codimension two in $\mathbb{P}^{n}$. Let $X, Y \in \mathcal{L}$ such that $h^{i}\left(\mathcal{I}_{X}(n)\right)=h^{i}\left(\mathcal{I}_{Y}(n)\right)$ for all $n \in \mathbb{Z}$ and $i \geq 0$. Then there exists an irreducible flat family of subschemes $\left\{X_{s}\right\}_{s \in S}$ in $\mathcal{L}$ with constant cohomology to which $X$ and $Y$ belong.

Proof. This is proved for even linkage classes of locally Cohen-Macaulay subschemes in [2, Proposition 3.1]. Since $h^{0}\left(\mathcal{I}_{X}(n)\right)=h^{0}\left(\mathcal{I}_{Y}(n)\right)$ for all $n$, we can find a graded free $S$-module $Q$ with surjections $Q \rightarrow I_{X}$ and $Q \rightarrow I_{Y}$. Sheafifying gives $\mathcal{E}$-type resolutions

$$
\begin{aligned}
0 & \rightarrow \mathcal{E}_{X} \rightarrow \mathcal{Q} \rightarrow \mathcal{I}_{X} \rightarrow 0 \\
0 & \rightarrow \mathcal{E}_{Y} \rightarrow \mathcal{Q} \rightarrow \mathcal{I}_{Y} \rightarrow 0
\end{aligned}
$$

The kernels $\mathcal{E}_{X}$ and $\mathcal{E}_{Y}$ are stably equivalent by Theorem 2.11 , so we may write $\mathcal{E}_{X} \oplus \mathcal{P}_{1} \cong \mathcal{E}_{Y}(d) \oplus \mathcal{P}_{2}$. We now consider two cases. If all the middle cohomology of both of these sheaves is zero, then $\mathcal{E}_{X}$ and $\mathcal{E}_{Y}$ are both dissocié, so the hypothesis 
that $h^{0}\left(\mathcal{I}_{X}(n)\right)=h^{0}\left(\mathcal{I}_{Y}(n)\right)$ for all $n$ shows that $\mathcal{E}_{X} \cong \mathcal{E}_{Y}$. In the case that some of the middle cohomology is nonzero, the fact that $h^{i}\left(\mathcal{I}_{X}(n)\right)=h^{i}\left(\mathcal{I}_{Y}(n)\right)$ for $1 \leq i<n-1$ shows that $d=0$. Now we can add $\mathcal{P}_{1}$ to the first sequence and $\mathcal{P}_{2}$ to the second sequence to get new $\mathcal{E}$-type resolutions in which the leftmost sheaves are isomorphic and the middle dissocié sheaves are $\mathcal{Q} \oplus \mathcal{P}_{1}$ and $\mathcal{Q} \oplus \mathcal{P}_{2}$. Since the cohomology of the middle sheaves must be the same and they are both dissocié, we see that they must be isomorphic. In either case, we obtain $\mathcal{E}$-type resolutions

$$
0 \rightarrow \mathcal{E} \rightarrow \mathcal{Q} \rightarrow \mathcal{O}
$$

for $X$ and $Y$ which involve the same sheaves (but possibly different maps).

Now let $U$ be the subset of $\mathbb{P}^{n}$ where $\mathcal{E}$ is locally free. By [6, Corollary 1.4], the codimension of the complement of $U$ is $\geq 3$. Let $V$ denote the parameter space for all homomorphisms $\phi: \mathcal{E} \rightarrow \mathcal{Q}$. Then if $p: \mathbb{P}^{n} \times V \rightarrow \mathbb{P}^{n}$ is the natural projection, there is a universal homomorphism $\phi: p^{*} \mathcal{E} \rightarrow p^{*} \mathcal{Q}$. On the open subset $U \times V \subset \mathbb{P}^{n} \times V$ we have the complex

$$
0 \rightarrow p^{*} \mathcal{E} \stackrel{\phi}{\longrightarrow} p^{*} \mathcal{Q} \stackrel{\Lambda^{r} \phi^{\vee} \otimes 1}{\longrightarrow} p^{*} \mathcal{O} \rightarrow 0
$$

If $j$ is the inclusion map for this open subset, applying $j_{*}$ extends the complex to all of $\mathbb{P}^{n} \times V$. By the preceding corollary, the set $V_{1}$ of $v \in V$ such that $0 \rightarrow p^{*} \mathcal{E} \otimes k(v) \rightarrow p^{*} \mathcal{Q} \otimes k(v) \rightarrow p^{*} \mathcal{O} \otimes k(v)$ is exact is open. Moreover, the morphisms corresponding to $X$ and $Y$ show that $V_{1}$ is nonempty. Since $V$ is smooth and irreducible, so is the nonempty open subset $V_{1} . V_{1}$ gives a family of subschemes $X_{v}$ with exact sequences

$$
0 \rightarrow \mathcal{E} \stackrel{\phi_{v}}{\longrightarrow} \mathcal{Q} \rightarrow \mathcal{O} \rightarrow \mathcal{O}_{X_{v}} \rightarrow 0
$$

which specialize to $X$ and $Y$. These subschemes are of pure codimension two by Lemma 3.3. Theorem 2.11 shows that they all lie in $\mathcal{L}$, which proves the statement of the lemma.

Definition 3.7. If $f: \mathbb{Z} \rightarrow \mathbb{N}$ is a function such that $f(n)=0$ for $n<<0$, then we define the function $f^{\#}$ by $f^{\#}(a)=\sum_{n \leq a} f(n)$.

Remark 3.8. We will be interested in comparing two such functions which have finite support and satisfy $\sum f(n)=\sum g(n)$. In this case, let $\left\{a_{i}\right\}$ and $\left\{b_{i}\right\}$ be the sequences of integers defined by the conditions $f^{\#}\left(a_{i}-1\right)<i \leq f^{\#}\left(a_{i}\right)$ and $g^{\#}\left(b_{i}-1\right)<i \leq g^{\#}\left(b_{i}\right)$. These are the unique nondecreasing sequences that satisfy $f(n)=\#\left\{i: a_{i}=n\right\}$ and $g(n)=\#\left\{i: b_{i}=n\right\}$. Note that $\#\left\{a_{i}\right\}=\sum f(n)=$ $\sum g(n)=\#\left\{b_{i}\right\}$. Now an elementary calculation shows that $f^{\#}(n) \geq g^{\#}(n) \Longleftrightarrow$ $a_{i} \leq b_{i}$ for each $i$. This characterization will be used in the proof of the next proposition.

Proposition 3.9. Let $X, Y \subset \mathbb{P}^{n}$ be subschemes of pure codimension two and let $h \geq 0$ be an integer. Then $X \leq_{h} Y$ if and only if there exist $\mathcal{N}$-type resolutions

$$
\begin{gathered}
0 \rightarrow \bigoplus \mathcal{O}(-n)^{r(n)} \rightarrow \mathcal{N} \rightarrow \mathcal{I}_{X}\left(h_{1}\right) \rightarrow 0, \\
0 \rightarrow \bigoplus \mathcal{O}(-n)^{s(n)} \rightarrow \mathcal{N} \rightarrow \mathcal{I}_{Y}\left(h_{2}\right) \rightarrow 0
\end{gathered}
$$

such that $h_{2}-h_{1}=h$ and $r^{\#} \geq s^{\#}$. 
Proof. First assume that $X \leq_{h} Y$, so that $Y$ is obtained from $X$ by a sequence of basic double links (which give $Z$ ) followed by a deformation. If $Z$ is obtained from $X$ by a basic double link of type $(s, h)$ and $X$ has an $\mathcal{N}$-type resolution

$$
0 \rightarrow \mathcal{P} \rightarrow \mathcal{N} \rightarrow \mathcal{I}_{X} \rightarrow 0
$$

then $Z$ has an $\mathcal{N}$-type resolution of the form

$$
0 \rightarrow \mathcal{P} \oplus \mathcal{O}(-s) \rightarrow \mathcal{N} \oplus \mathcal{O}(-s+h) \rightarrow \mathcal{I}_{Z}(h) \rightarrow 0
$$

(this can be seen by applying Proposition 1.11 and Proposition 1.8 and splitting off a line bundle summand). If we add the summand $\mathcal{O}(-s+h)$ to both terms in the resolution for $\mathcal{I}_{X}$, we see that the dissocié part becomes $\mathcal{P} \oplus \mathcal{O}(-s+h)$. Comparing this to $\mathcal{P} \oplus \mathcal{O}(-s)$, we find that $r^{\#} \geq s^{\#}$ for this case. The same argument shows that if we do a sequence of basic double links to get from $X$ to $Z$, then we can find such resolutions of $X$ and $Z$.

Let such a pair of resolutions be

$$
\begin{gathered}
0 \rightarrow \bigoplus \mathcal{O}(-n)^{r(n)} \rightarrow \mathcal{N} \rightarrow \mathcal{I}_{X} \rightarrow 0 \\
0 \rightarrow \bigoplus \mathcal{O}(-n)^{s(n)} \rightarrow \mathcal{N} \rightarrow \mathcal{I}_{Z}(h) \rightarrow 0
\end{gathered}
$$

with $r^{\#} \geq s^{\#}$. Let $Y$ be obtained from $Z$ by a deformation which preserves cohomology and even linkage class. Since $Y$ is in the same even linkage class as $X$, we can obtain $Y$ from $X$ by an even sequence of links. Applying the mapping cone procedure, we get a resolution for $Y$ of the form

$$
0 \rightarrow \mathcal{P} \rightarrow \mathcal{N} \oplus \mathcal{Q} \rightarrow \mathcal{I}_{Y}(h) \rightarrow 0
$$

where $\mathcal{P}, \mathcal{Q}$ are dissocié (if some of the middle cohomology of $\mathcal{N}$ is nonzero, it's clear that the twist $h$ is the same for both $Z$ and $Y$. If all the middle cohomology vanishes, then $\mathcal{N}$ is dissocié and it's also clear that we can use the same twist $h$ for both $Z$ and $Y$ ). Add the dissocié sheaf $\mathcal{Q}$ to the $\mathcal{N}$-type resolutions for $X$ and $Z$ above. With these new resolutions, we still have the inequalities $r_{1}^{\#} \geq s_{1}^{\#}$, where $\bigoplus \mathcal{O}(-n)^{s_{1}(n)} \cong \bigoplus \mathcal{O}(-n)^{s(n)} \bigoplus \mathcal{Q}$ and $\bigoplus \mathcal{O}(-n)^{r_{1}(n)} \cong \bigoplus \mathcal{O}(-n)^{r(n)} \oplus \mathcal{Q}$. Further, the fact that the cohomology of $Y$ and the cohomology of $Z$ are the same shows that $\mathcal{P} \cong \bigoplus \mathcal{O}(-n)^{s_{1}(n)}$. This proves that there exist $\mathcal{N}$-type resolutions as in the proposition.

Now assume that $X$ and $Y$ have such $\mathcal{N}$-type resolutions. We wish to show that $X \leq_{h} Y$. This is essentially [10, IV, Lemma 5.2]. We give a slightly different proof here, based on the argument of [1, Theorem 2.4]. Rewrite $\bigoplus \mathcal{O}(-n)^{r(n)}=$ $\bigoplus \mathcal{O}\left(-a_{i}\right)$ and $\bigoplus \mathcal{O}(-n)^{s(n)}=\bigoplus \mathcal{O}\left(-b_{i}\right)$ with the $a_{i}$ and $b_{i}$ nondecreasing. By Remark 3.8, we have that $a_{i} \leq b_{i} \Longleftrightarrow r^{\#} \geq s^{\#}$. By looking at Chern classes, we can see that $\sum b_{i}-\sum a_{i}=h_{2}-h_{1}=h$. I'll first show by induction on $h$ that there exists a scheme $Y^{\prime}$ which is obtained from $X$ by a sequence of basic double links with heights summing to $h$ which has the same cohomology as $Y$. For the induction base case $h=0$ we have $a_{i}=b_{i}$ for all $i$ and hence $r=s$. Here the exact 
sequences show that $h^{i}\left(\mathcal{I}_{X}(n)\right)=h^{i}\left(\mathcal{I}_{Y}(n)\right)$ for all $n$ and $i \geq 0$, so we may choose $Y^{\prime}=X$

Now consider the case where $h>0$. Let $n=\max \left\{i: b_{i}>a_{i}\right\}$ so that $a_{i}=b_{i}$ for $i>n$. We claim that $h^{0}\left(\mathcal{I}_{X}\left(h_{1}+n\right)\right) \neq 0$. From the exact sequence for $\mathcal{I}_{X}$, we see that

$$
h^{0}\left(\mathcal{I}_{X}\left(h_{1}+n\right)\right)=h^{0}(\mathcal{N}(n))-\sum_{a_{i} \leq n} h^{0}\left(\mathcal{O}\left(n-a_{i}\right)\right)
$$

but this is strictly greater than

$$
h^{0}(\mathcal{N}(n))-\sum_{b_{i} \leq n} h^{0}\left(\mathcal{O}\left(n-b_{i}\right)\right)=h^{0}\left(\mathcal{I}_{Y}\left(h_{2}+n\right)\right) \geq 0
$$

and this proves the claim. This allows us to obtain a new scheme $X_{1}$ from $X$ by a basic double link of type $\left(h_{1}+n, b_{n}-a_{n}\right)$. $X_{1}$ has a resolution of the form

$$
0 \rightarrow \bigoplus \mathcal{O}\left(-a_{i}\right) \oplus \mathcal{O}\left(-b_{n}\right) \rightarrow \mathcal{N} \oplus \mathcal{O}\left(-a_{n}\right) \rightarrow \mathcal{I}_{X_{1}}\left(h_{1}+b_{n}-a_{n}\right) \rightarrow 0
$$

Adding an extra line bundle summand $\mathcal{O}\left(-a_{n}\right)$ to the resolution for $Y$ we get

$$
0 \rightarrow \bigoplus \mathcal{O}\left(-b_{i}\right) \oplus \mathcal{O}\left(-a_{n}\right) \rightarrow \mathcal{N} \oplus \mathcal{O}\left(-a_{n}\right) \rightarrow \mathcal{I}_{Y}\left(h_{2}\right) \rightarrow 0
$$

Reordering the summands of these, we find that the inequalities still hold, and summing the differences gives that the new value of $h$ is $h-b_{n}+a_{n}<h$. Thus we can apply the induction hypothesis to obtain a subscheme $Y^{\prime}$ which is obtained by a sequence of basic double links from $X_{1}$ with heights summing to $h-b_{n}+a_{n}$ and has the same cohomology $Y$. It follows that $Y^{\prime}$ can be obtained from $X$ by a sequence of basic double links with heights summing to $h$. Now we can apply Lemma 3.6 to see that $Y^{\prime}$ can be deformed with constant cohomology and even linkage class to $Y$. Thus $X \leq_{h} Y$.

Corollary 3.10. Domination is transitive. In particular, it defines a partial ordering on an even linkage class $\mathcal{L}$.

Proof. If $X \leq Y$ and $Y \leq Z$, then we can find $\mathcal{N}$-type resolutions as in Proposition 3.9. Using the stable equivalence of the reflexive sheaves in the middle, we can add line bundle summands to the three resolutions to obtain exact sequences

$$
\begin{gathered}
0 \rightarrow \bigoplus \mathcal{O}(-n)^{r(n)} \rightarrow \mathcal{N} \rightarrow \mathcal{I}_{X} \rightarrow 0 \\
0 \rightarrow \bigoplus \mathcal{O}(-n)^{s(n)} \rightarrow \mathcal{N} \rightarrow \mathcal{I}_{Y}\left(h_{1}\right) \rightarrow 0 \\
0 \rightarrow \bigoplus \mathcal{O}(-n)^{t(n)} \rightarrow \mathcal{N} \rightarrow \mathcal{I}_{Z}\left(h_{2}\right) \rightarrow 0
\end{gathered}
$$

with $r^{\#} \geq s^{\#}$ and $s^{\#} \geq t^{\#}$. It follows that $r^{\#} \geq t^{\#}$, so Proposition 3.9 shows that $X \leq Z$. 
Definition 3.11. Let $\mathcal{L}$ be an even linkage class of subschemes of pure codimension two in $\mathbb{P}^{n}$. We say that $\mathcal{L}$ has the Lazarsfeld-Rao property if $\mathcal{L}$ has a minimal element with respect to domination.

Remark 3.12. In view of the definition of domination and the fact that domination is a partial order, this definition is equivalent to the usual definition used in [10] or $[2]$.

Remark 3.13. In view of Proposition 3.9 we can try to produce minimal subschemes for an even linkage class $\mathcal{L}$ by finding injections $\bigoplus \mathcal{O}(-n)^{r(n)} \hookrightarrow \mathcal{N}$ whose cokernel is a twisted ideal sheaf of a subscheme of pure codimension two $(\mathcal{N}$ is in the stable equivalence class corresponding to the $\mathcal{N}$-type resolutions) such that the function $r^{\#}$ is as large as possible. Specifically, it would be ideal to have a function $q$ associated to the reflexive sheaf $\mathcal{N}$ such that there exists an injection $\bigoplus \mathcal{O}(-n)^{q(n)} \rightarrow \mathcal{N}$ as above and has the property that if $\bigoplus \mathcal{O}(-n)^{r(n)} \rightarrow \mathcal{N}$ is another such injection, then $r^{\#}(n) \leq q^{\#}(n)$ for each $n \in \mathbb{Z}$. Martin-Deschamps and Perrin have already carried out this work in the case when $\mathcal{N}$ is a vector bundle on $\mathbb{P}^{3}$. Fortunately, their results about injections $\bigoplus \mathcal{O}(-n)^{r(n)} \hookrightarrow \mathcal{N}$ are stated for the case when $\mathcal{N}$ is only a torsion free sheaf on $\mathbb{P}^{3}$ and their proofs work on $\mathbb{P}^{n}$. In particular, their solution applies to the reflexive sheaves $\mathcal{N}$ that come from $\mathcal{N}$-type resolutions. What follows is a brief summary of their results, stated in the generality in which their proofs hold.

Definition 3.14. Let $B \subset A$ be an inclusion of $\mathcal{O}$-modules. We say that $B$ is maximal in $A$ if for each $\mathcal{O}$-module $B^{\prime}$ having the same rank as $B$ and satisfying $B \subset B^{\prime} \subset A$ we have $B=B^{\prime}$.

Proposition 3.15. Let $B \subset A$ be an inclusion of $\mathcal{O}$-modules, where $B$ is locally free and $A$ is torsion free. Then the following are equivalent:

1. $B$ is maximal in $A$.

2. $A / B$ is torsion free.

3. $A / B$ is torsion free in codimension one.

4. $A / B$ is locally free in codimension one.

5. $A / B$ has constant rank in codimension one.

6. $A / B$ is locally a direct factor of $A$ in codimension one.

Proof. See [10, IV, Proposition 1.2]. In fact, their proof shows that these are equivalent if $B$ is only reflexive.

Definition 3.16. Let $\mathcal{N}$ be a torsion free sheaf on $\mathbb{P}^{n}$. Let $\bigoplus S(-n)^{l(n)} \rightarrow H_{*}^{0}(\mathcal{N})$ be a minimal graded surjection of $S$-modules. This defines the function $l_{\mathcal{N}}=l$. Sheafifying this surjection defines the map $\sigma: \bigoplus \mathcal{O}(-n)^{l(n)} \rightarrow \mathcal{N}$. For $a \in \mathbb{Z}$, the map $\sigma_{a}$ is defined by restricting $\sigma$ to $\bigoplus_{n \leq a} \mathcal{O}(-n)^{l(n)}$.

Remark 3.17. For each $a \in \mathbb{Z}, \sigma_{a}$ is well-defined up to a choice of basis for $\bigoplus_{n \leq a} \mathcal{O}(-n)^{l(n)}$ and hence the image of $\sigma_{a}$ (which is denoted by $\mathcal{N}_{\leq a}$ in [10]) depends only on $a$. The function $l=l_{\mathcal{N}}$ could have been defined by the formula $l(n)=\operatorname{dim}_{k}\left(N \otimes_{S} k\right)_{n}$, where $N=H_{*}^{0}(\mathcal{N})$.

Definition 3.18. Let $\mathcal{N}$ be a torsion free sheaf on $\mathbb{P}^{n}$ and let $\sigma_{a}$ be as in definition 3.16. Then we define

$$
\alpha_{a}=\operatorname{rank}\left(\sigma_{a}\right)
$$




$$
\beta_{a}=\inf _{D}\left(\operatorname{rank}\left(\left.\sigma_{a}\right|_{D}\right)\right)
$$

where this infimum is taken over all integral divisors $D$. We always have the inequalities

$$
0 \leq \beta_{a} \leq \alpha_{a} \leq l^{\#}(a)
$$

and we define $a_{0}=\sup \left\{a \in \mathbb{Z}: \alpha_{a}=\beta_{a}=l^{\#}(a)\right\}$ if this exists, otherwise $a_{0}=\infty$. We also define $a_{1}=\inf \left\{a \in \mathbb{Z}: \alpha_{a}=\beta_{a}=\operatorname{rank}(\mathcal{N})\right\}$.

Definition 3.19. Let $\mathcal{N}$ be a torsion free sheaf on $\mathbb{P}^{n}$. Then we define the function $q=q_{\mathcal{N}}$ by

1. $q(n)=l(n)$ if $n<a_{0}$,

2. $q^{\#}(n)=\inf \left\{\alpha_{n}-1, \beta_{n}\right\}$ otherwise.

Remark 3.20. This is the function referred to in Remark 3.13. It is a consequence of the results which follow that if $\mathcal{N}$ is reflexive with $\mathcal{E} x t^{1}(\mathcal{N}, \mathcal{O})=0$ and $H_{*}^{1}\left(\mathcal{N}^{\vee}\right)=0$, then there is an injection $\bigoplus \mathcal{O}(-n)^{q(n)} \rightarrow \mathcal{N}$ whose cokernel is the twisted ideal sheaf of a subscheme of pure codimension two. Further, for any other such injection $\bigoplus \mathcal{O}(-n)^{r(n)} \rightarrow \mathcal{N}$ we have that $r^{\#}(n) \leq q^{\#}(n)$ for all $n \in \mathbb{Z}$.

Proposition 3.21. Let $\mathcal{N}$ be a torsion free sheaf on $\mathbb{P}^{n}$ with associated functions $l, q, a_{0}$ and $a_{1}$ as in the preceding definitions.

1. If $\mathcal{N}$ is dissocie, then $a_{0}=\infty, q=l$ and $q^{\#}(\infty)=\operatorname{rank}(\mathcal{N})$.

2. If $\mathcal{N}$ is not dissocié, then $q^{\#}\left(a_{1}\right)=q^{\#}(\infty)=\operatorname{rank}(\mathcal{N})-1,0 \leq q(n) \leq l(n)$ for each $n \in \mathbb{Z}, a_{0} \leq a_{1}<\infty, q\left(a_{0}\right)<l\left(a_{0}\right)$ and $q(n)=0$ for $n>a_{1}$.

Proof. See [10, IV, Proposition 2.7].

Proposition 3.22. Let $\mathcal{N}$ be a torsion free sheaf on $\mathbb{P}^{n}$ and suppose that $\mathcal{N}^{\prime}=$ $\mathcal{N} \oplus \mathcal{P}$ where $\mathcal{P}$ is dissocié. Then $q_{\mathcal{N}^{\prime}}=q_{\mathcal{N}}+l_{\mathcal{P}}$.

Proof. See [10, IV, Proposition 2.9].

Theorem 3.23. Let $\mathcal{N}$ be a torsion free sheaf on $\mathbb{P}^{n}$ with associated function $q=$ $q_{\mathcal{N}}$. If an injection $\bigoplus \mathcal{O}(-n)^{r(n)} \hookrightarrow \mathcal{N}$ has image maximal in $\mathcal{N}$, then $r^{\#} \leq q^{\#}$.

Proof. See [10, IV, theorem 3.1].

Theorem 3.24. Let $\mathcal{N}$ be a torsion free sheaf on $\mathbb{P}^{n}$ with associated function $q=q_{\mathcal{N}}$ and $\sigma: \bigoplus \mathcal{O}(-n)^{l(n)} \rightarrow \mathcal{N}$. Then $\bigoplus \mathcal{O}(-n)^{l(n)}$ has a direct factor of the form $\bigoplus \mathcal{O}(-n)^{q(n)}$ whose image under $\sigma$ is maximal in $\mathcal{N}$. Conversely, if $\bigoplus \mathcal{O}(-n)^{q(n)} \stackrel{\beta}{\rightarrow} \mathcal{N}$ is injective with maximal image, then there is a split injection $\tau: \bigoplus \mathcal{O}(-n)^{q(n)} \rightarrow \bigoplus \mathcal{O}(-n)^{l(n)}$ such that $\beta=\sigma \circ \tau$.

Proof. For the first part, see [10, IV, Theorem 3.4]. The converse part is the second part of [10, IV, Theorem 3.7].

Proposition 3.25. Let $\mathcal{N}$ be a torsion free sheaf on $\mathbb{P}^{n}$ with associated function $q=q_{\mathcal{N}}$ and let $\mathcal{P}$ be dissocié. If $\tau: \bigoplus \mathcal{O}(-n)^{q(n)} \oplus \mathcal{P} \rightarrow \mathcal{N} \oplus \mathcal{P}$ is an injection with maximal image, then there is an automorphism $\nu$ of $\oplus \mathcal{O}(-n)^{q(n)} \oplus \mathcal{P}$ such that the diagram

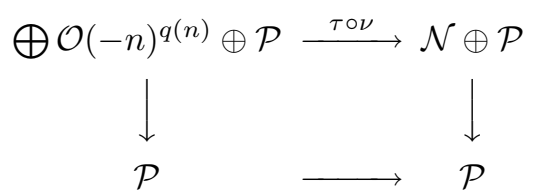


commutes, where the vertical arrows are the canonical projections.

Proof. See [10, IV, Proposition 3.9].

Theorem 3.26. Let $\mathcal{L}$ be an even linkage class corresponding to the stable equivalence class $\left[\mathcal{N}_{0}\right]$ via $\mathcal{N}$-type resolutions. Assume $\mathcal{N}_{0} \neq 0$ and let $q=q_{\mathcal{N}_{0}}$ be the associated function. Then there exists $X_{0} \in \mathcal{L}$ having an $\mathcal{N}$-type resolution of the form

$$
0 \rightarrow \bigoplus \mathcal{O}(-n)^{q(n)} \rightarrow \mathcal{N}_{0} \rightarrow \mathcal{I}_{X_{0}}(h) \rightarrow 0
$$

and satisfying $X_{0} \leq Y$ for each $Y \in \mathcal{L}$. In particular, $\mathcal{L}$ has the Lazarsfeld-Rao property. $X_{0}^{\prime}$ is another such minimal element of $\mathcal{L} \Longleftrightarrow X_{0}^{\prime}$ has a resolution of the same form $\Longleftrightarrow X_{0}$ deforms to $X_{0}^{\prime}$ with constant cohomology through subschemes in $\mathcal{L}$.

Proof. This statement for locally Cohen-Macaulay curves in $\mathbb{P}^{3}$ is contained in Propositions 4.4 and 4.5 of $[10, \mathrm{IV}]$. There exists an injective homomorphism $\tau: \bigoplus \mathcal{O}(-n)^{q(n)} \rightarrow \mathcal{N}_{0}$ whose image is maximal in $\mathcal{N}_{0}$ by Theorem 3.24. From Proposition 3.21 we have that $q^{\#}\left(a_{1}\right)=\operatorname{rank}\left(\mathcal{N}_{0}\right)-1$, so the cokernel of $\tau$ has rank one. By maximality, this cokernel is torsion free, hence it is a twisted ideal sheaf of a subscheme in $\mathbb{P}^{n}$ of pure codimension two by Lemma 3.3 (after we twist the morphism until the degrees become equal, condition (B) of Lemma 3.3 holds). Let this subscheme be $X_{0}$.

Now let $Y \in \mathcal{L}$. Then $Y$ has an $\mathcal{N}$-type resolution of the form

$$
0 \rightarrow \bigoplus \mathcal{O}(-n)^{r(n)} \stackrel{\nu}{\rightarrow} \mathcal{N}_{0} \oplus \mathcal{Q} \rightarrow \mathcal{I}_{Y}(k) \rightarrow 0
$$

with $\mathcal{Q}$ dissocié. Since $\mathcal{I}_{Y}(k)$ is torsion free, the image of $\nu$ is maximal in $\mathcal{N}_{0} \oplus \mathcal{Q}$. It follows from Theorem 3.23 that if $q_{1}$ is the function associated to $\mathcal{N}_{0} \oplus \mathcal{Q}$, then we have $r^{\#} \leq q_{1}^{\#}$. On the other hand, if we add the trivial summand $\mathcal{Q}$ on to the resolution for $X_{0}$ and apply Proposition 3.22, the resolution for $X_{0}$ corresponds to this function $q_{1}$. In view of Proposition 3.9 and Remark 3.20, we see that $X_{0} \leq Y$.

Finally, let $X_{0}^{\prime}$ be another subscheme for $\mathcal{L}$. If $X_{0}^{\prime}$ has a resolution of the form that $X_{0}$ does, then $h^{i}\left(\mathcal{I}_{X_{0}^{\prime}}(n)\right)=h^{i}\left(\mathcal{I}_{X_{0}}(n)\right)$ for all $n, i$, and hence by Lemma 3.6 we see that $X_{0}$ can be deformed to $X_{0}^{\prime}$ with constant cohomology and even linkage class. If there exists such a deformation, then by definition of $\leq$ we have that $X_{0}^{\prime} \leq_{0} X_{0}$, hence $X_{0}^{\prime}$ is also minimal for $\mathcal{L}$. It remains to be shown that if $X_{0}^{\prime}$ is minimal, then it has a resolution of the same form that $X_{0}$ does.

If we write an $\mathcal{N}$-type resolution for $X_{0}^{\prime}$ as

$$
0 \rightarrow \bigoplus \mathcal{O}(-n)^{r(n)} \rightarrow \mathcal{N}_{0} \oplus \mathcal{Q} \rightarrow \mathcal{I}_{X_{0}^{\prime}}(l) \rightarrow 0
$$

and add the trivial summand $\mathcal{Q}$ to the resolution for $X_{0}$, we conclude that $q_{1}^{\#} \leq r^{\#}$ by minimality of $X_{0}^{\prime}$ and that $r^{\#} \leq q_{1}^{\#}$ from Theorem 3.23. It follows that $q_{1}^{\#}=r^{\#}$. Since these are both functions of finite support, we see that $q_{1}=r$. Applying Proposition 3.22, we have that $r=q+l_{\mathcal{Q}}$. Now we apply Proposition 3.25 to change the map so that the image in $\mathcal{N}_{0} \oplus \mathcal{Q}$ is the same, but in such a way that the summand $\mathcal{Q}$ can be split off. Splitting off $\mathcal{Q}$, we get a resolution

$$
0 \rightarrow \bigoplus \mathcal{O}(-n)^{q(n)} \rightarrow \mathcal{N}_{0} \rightarrow \mathcal{I}_{X_{0}^{\prime}} \rightarrow 0
$$

This completes the proof. 
Remark 3.27. If $X_{0}$ is minimal for $\mathcal{L}$ as in the above theorem, then there exists an exact sequence

$$
0 \rightarrow \bigoplus \mathcal{O}(-n)^{q(n)} \rightarrow \mathcal{N} \rightarrow \mathcal{I}_{X_{0}}(h) \rightarrow 0 .
$$

Since $q(n)=l(n)$ for $n<a_{0}$ and $q\left(a_{0}\right)<l\left(a_{0}\right)$ by Proposition 3.21, we have that

$$
s\left(X_{0}\right)=\min \left\{n: h^{0}\left(\mathcal{I}_{X_{0}}(n)\right) \neq 0\right\}=a_{0}+h .
$$

Proposition 3.28. Let $\mathcal{L}$ be the even linkage class corresponding to the stable equivalence class $\left[\mathcal{N}_{0}\right]$ via $\mathcal{N}$-type resolution, with $\mathcal{N}_{0} \neq 0$. Let $\sigma: \bigoplus \mathcal{O}(-n)^{l(n)} \rightarrow$ $\mathcal{N}_{0}$ be the surjection of Definition 3.16 and let $\mathcal{E}_{0}$ denote the kernel of $\sigma$. Then $X_{0}$ is minimal for $\mathcal{L} \Longleftrightarrow X_{0}$ has an $\mathcal{E}$-type resolution of the form

$$
0 \rightarrow \mathcal{E}_{0} \rightarrow \bigoplus \mathcal{O}(-n)^{l(n)-q(n)} \rightarrow \mathcal{I}_{X_{0}}(h) \rightarrow 0
$$

Proof. This statement for locally Cohen-Macaulay curves in $\mathbb{P}^{3}$ is contained in Propositions 4.4 and 4.5 of [10, IV]. Theorem 3.26 gives an $\mathcal{N}$-type resolution for the minimal subscheme $X_{0}$. By the second part of Theorem 3.24 the corresponding map $\bigoplus \mathcal{O}(-n)^{q(n)} \hookrightarrow \mathcal{N}_{0}$ factors through $\sigma$ as a direct factor of $\bigoplus \mathcal{O}(-n)^{l(n)}$. It follows that we have a commutative diagram

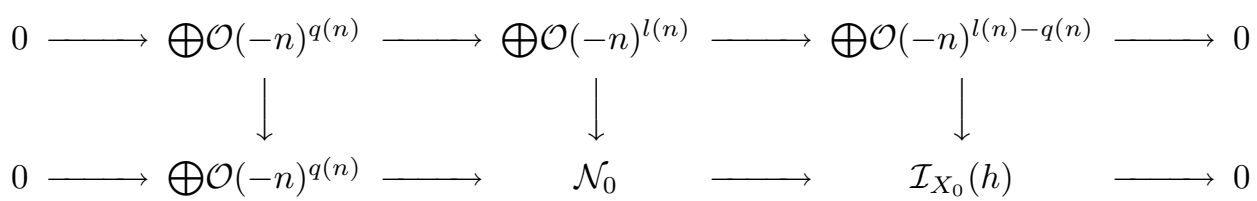

whose rows are exact sequences and the leftmost vertical map is an isomorphism. Since the middle map is surjective with kernel $\mathcal{E}_{0}$, the same holds for the rightmost map by the snake lemma, which gives the resolution. The map $H_{*}^{0}\left(\bigoplus \mathcal{O}(-n)^{l(n)}\right) \rightarrow$ $H_{*}^{0}\left(I_{X_{0}}(h)\right)$ is surjective because $H_{*}^{0}\left(\bigoplus \mathcal{O}(-n)^{l(n)}\right) \rightarrow H_{*}^{0}\left(\mathcal{I}_{X_{0}}(h)\right)$ is, hence it is an $\mathcal{E}$-type resolution.

Conversely, if $X_{0}^{\prime}$ has such a resolution, it is in the same even linkage class as some minimal subscheme $X_{0}$ for $\mathcal{L}$ by Theorem 2.11 and this resolution shows that $X_{0}^{\prime}$ has the same cohomology as $X_{0}$. Applying Lemma 3.6 we see that $X_{0}$ can be deformed with constant cohomology and even linkage class to $X_{0}^{\prime}$, hence $X_{0}^{\prime}$ is also minimal by Theorem 3.26.

Proposition 3.29. Let $\mathcal{E}_{0}$ be the kernel of the map $\sigma: \bigoplus \mathcal{O}(-n)^{l(n)} \rightarrow \mathcal{N}_{0}$ as in Proposition 3.28. Let $q$ be the function associated to $\mathcal{N}_{0}$ and let $q^{\prime}$ be the function associated to $\mathcal{E}_{0}^{\vee}$. Then $q$ and $q^{\prime}$ are related by the formula

$$
q(n)+q^{\prime}(n)=l(n)-\epsilon_{0}(n)-\epsilon_{1}(n)
$$

where $\epsilon_{0}\left(a_{0}\right)=1, \epsilon_{0}(n)=0$ for $n \neq 0, \epsilon_{1}\left(a_{1}\right)=1$, and $\epsilon_{1}(n)=0$ for $n \neq a_{1}$.

Proof. See [10, IV, Proposition 5.7]. 
Theorem 3.30. Let $\mathcal{L}$ be an even linkage class corresponding to the stable equivalence class $\left[\mathcal{N}_{0}\right]$ via $\mathcal{N}$-type resolution, with $\mathcal{N}_{0} \neq 0$. Let $X_{0}$ be a minimal subscheme for $\mathcal{L}$ having $\mathcal{N}$-type resolution

$$
0 \rightarrow \bigoplus \mathcal{O}(-n)^{q(n)} \rightarrow \mathcal{N}_{0} \rightarrow \mathcal{I}_{X_{0}}(h) \rightarrow 0
$$

Then there exist hypersurfaces of degrees $a_{0}+h$ and $a_{1}+h$ which contain $X_{0}$ and link $X_{0}$ to a subscheme $Y_{0}$ which is minimal for its even linkage class.

Proof. This was first proved for curves in $\mathbb{P}^{3}$ in [10, IV, Theorem 5.10]. By definition of $a_{1}$, the morphism $\sigma_{a_{1}}$ is surjective on an open set which contains the generic points of each integral divisor in $\mathbb{P}^{n}$, hence this also holds for the induced map $\bigoplus_{n \leq a_{1}} \mathcal{O}(-n)^{l(n)-q(n)} \rightarrow \mathcal{I}_{X_{0}}(h)$. Since the minimal value of $n$ making $l(n)-$ $q(n) \neq 0$ is $n=a_{0}$, it follows that this yields hypersurfaces of degrees varying from $a_{0}+h$ to $a_{1}+h$ which cut out a subscheme of codimension $>1$. It follows that we can find hypersurfaces of degrees $a_{0}+h$ and $a_{1}+h$ which meet properly, and hence link $X_{0}$ to another subscheme $Y_{0}$.

Applying Proposition 1.8 we obtain a resolution for $Y_{0}$ of the form

$$
0 \rightarrow \bigoplus \mathcal{O}(n)^{l(n)-q(n)} \rightarrow \mathcal{E}_{0}^{\vee} \oplus \mathcal{O}\left(-a_{0}\right) \oplus \mathcal{O}\left(-a_{1}\right) \rightarrow \mathcal{I}_{Y_{0}}\left(a_{0}+a_{1}+h\right) \rightarrow 0
$$

Proposition 3.29 shows that if $\mathcal{M}=\mathcal{E}_{0}^{\vee} \oplus \mathcal{O}\left(-a_{0}\right) \oplus \mathcal{O}\left(-a_{1}\right)$, then $q_{\mathcal{M}}(n)=l(n)-$ $q(n)$ for each $n \in \mathbb{Z}$. It follows from this resolution and Theorem 3.26 that $Y_{0}$ is minimal for its even linkage class.

\section{REFERENCES}

1. E. Ballico, G. Bolondi and J. Migliore, The Lazarsfeld-Rao problem for liaison classes of two-codimensional subschemes of $\mathbb{P}^{n}$, Amer J. Math. 113 (1991), 117-128. MR 92c:14047

2. G. Bolondi and J. Migliore, The Structure of an Even Liaison Class, Trans. Amer. Math. Soc. 316 (1989), 1-37. MR 90b:14060

3. A. Grothendieck, Eléments de Géométrie Algébrique IV, Inst. Hautes Études Sci. Publ. Math. 28 (1966). MR 36:178

4. R. Hartshorne, Ample Subvarieties of Algebraic Varieties, Lecture Notes in Math., vol. 156, Springer, 1970. MR 44:211

5. R. Hartshorne, Algebraic Geometry, Springer-Verlag, Berlin, Heidelberg and New York, 1977. MR 57:3116

6. R. Hartshorne, Stable Reflexive Sheaves, Math. Ann. 254 (1980), 121-176. MR 82b:14011

7. R. Hartshorne, Generalized Divisors on Gorenstein Schemes, K-Theory 8 (1994), 287-339. CMP 94:17

8. S. Kleiman, Geometry on Grassmanians and applications to splitting bundles and smoothing cycles, Inst. Hautes Études Sci. Publ. Math. 36 (1969), 281-298. MR 42:281

9. R. Lazarsfeld and A. P. Rao, Linkage of General Curves of Large Degree, Lecture Notes in Math., vol. 997, Springer, 1983, pp. 267-289. MR 85d:14043

10. M. Martin-Deschamps and D. Perrin, Sur la Classification des Courbes Gauches, Astérisque 184-185, 1990. MR 91h:14039

11. M. Martin-Deschamps and D. Perrin, Construction de Courbes Lisses: un Théorème de Bertini, vol. 22, Laboratoire de Mathématiques de l'Ecole Normale Supérieure, 1992.

12. Hideyuki Matsumura, Commutative Ring Theory, Cambridge University Press, 1986. MR 88h: 13001

13. J. Migliore, An Introduction to Deficiency Modules and Liaison Theory for Subschemes of Projective Space, Global Analysis Research Center, Seoul National University, Lecture Notes Series 24 (1994). CMP 95:03 
14. C. Peskine and L. Szpiro, Liaison des variétés algébriques, I, Inventiones Math. 26 (1972), 271-302. MR 51:526

15. A. P. Rao, Liaison Among Curves in $\mathbb{P}^{3}$, Inventiones Math. 50 (1979), 205-217. MR 80e: 14023

16. A. P. Rao, Liaison Equivalence Classes, Math. Ann. 258 (1981), 169-173. MR 83j:14045

2919 Fulton St., Berkeley, California 94705

E-mail address: nollet@math.berkeley.edu 